\section{Dynamic plastic response of beams subjected to localised pulse loads}

\author{
Nicolas Thil', Navid Mehreganian², \\ Mojtaba Moatamedi ${ }^{3}$, Luke A Louca ${ }^{2}$ and \\ Arash Soleiman Fallah ${ }^{1,4}$
}

International Journal of Protective Structures

$|-3|$

(C) The Author(s) 2018

Article reuse guidelines:

sagepub.com/journals-permissions DOI: |0.||77/204|4|96|880772|

journals.sagepub.com/home/prs

@SAGE

\begin{abstract}
Localised blast loads give rise to high gradients of overpressure detrimental to structural elements as beams and plates. This article presents an analytical study on the dynamic plastic response of beams made of a ductile metallic material due to close-in pulse pressure loading. The close-in pressure load is characterised by a spatially varying function constant over a central region and exponentially decaying beyond it. The temporal pulse shape is assumed to take different forms. The exact static plastic collapse load was obtained for the characteristic load using the framework of plastic limit analysis, whereby the analysis was then extended to the dynamic case by considering the appropriate yield surface and inclusion of inertia forces. The yield surfaces considered were representative of pure bending, the interactions between the bending moment and transverse shear, and bending moment and membrane force, each corresponding to a special case depending on the geometry of the beam. A time-dependent, kinematically admissible velocity profile was assumed to treat the dynamic formulations in interaction of each phenomenon. A study on the strain-rate sensitivity was also presented, and existence of a critical pressure triggering the apparition of travelling plastic hinges was hence highlighted. For blast loads of high magnitude, the expressions for normalised deflection were furnished in terms of the impulsive velocity. The analytical models were validated by performing a parametric study on the two-dimensional representative of the beam model in commercial finite element software ABAQUS 6.14. The numerical results show a good correlation with the analytical results in each case.
\end{abstract}

\title{
Keywords
}

Localised blast, rigid plastic beam, bound theorems of plasticity, travelling plastic hinge, dynamic plastic response

\footnotetext{
'Department of Aeronautics, Imperial College London, London, UK

${ }^{2}$ Department of Civil \& Environmental Engineering, Imperial College London, London, UK

${ }^{3}$ Faculty of Engineering Science and Technology, UiT The Arctic University of Norway, Narvik, Norway

${ }^{4}$ Department of Mechanical, Aerospace and Civil Engineering, Brunel University London, London, UK
}

\section{Corresponding author:}

Arash Soleiman Fallah, Department of Aeronautics, Imperial College London, City and Guilds Building, South Kensington Campus, London SW7 2AZ, UK.

Email: as3@imperial.ac.uk 


\section{Introduction}

Close-in charges, such as those of improvised explosive devices (IEDs), induce a localised blast load which can potentially lead to structural failure, detrimental damage to critical equipment and loss of life. Assessment of the response of protective systems to such blasts is, therefore, a major task in the fields of aeronautics, civil, offshore and military engineering; for which many mitigative measures have been proposed in the literature. Some measures included the design of cost-effective mine-field clearing equipment, such as tumbleweed minesweeper (Webster, 2013), while others have focused on investigating the impact of blast load parameters such as load duration, standoff distance, pulse shape and impulse, by the virtue of experimental or numerical techniques (Jacob et al., 2007; Larcher and Casadei, 2010; Yuen and Nurick, 2001, 2005). The primary structural components investigated in these studies were beams and plates, most commonly made of steel or alloys of steel, due to their significant post-yield deformation capacity to sustain extreme loads and absorb significant amounts of energy.

There are experimental studies on the influence of material type, geometry and boundary conditions on the response of the structural systems (Bonorchis and Nurick, 2007; Jacob et al., 2004; Langdon et al., 2015; Nurick and Balden, 2010; Wierzbicki and Nurick, 1996) to such blast loads. Other examples include the work of Karagiozova et al. (2013) on the response of hollow beams where the deformation of the cross-section and subsequent ovalisation helps dissipate the kinetic energy of the impact. In the similar spirit, Chung Kim Yuen et al. (2012) studied the V-shaped plates and deduced the angle that could optimise the deflection of the blast wave on the element surface. The influence of the structural member's material was also considered.

Although such experiments provide invaluable insight into the influence of parameters involved in the response parameters, the repetitive experimental blast tests can rapidly become prohibitively expensive as the number of parameters involved increase. Besides, many other parameters could influence the behaviour of a beam under localised blast, namely, the distribution of the pressure, the transverse shear effect, membrane forces and the strain-rate sensitivity phenomenon observed in the material. The plethora of these variables necessitates the designer to rely on the analytical methods and on the dimensionless analyses to predict the performance of the beams without the necessity of conducting experiments in every case. In fact, since the pioneering works of Jones (1990), Symonds and Yu (1985) and Taylor (1963), other analytical models have been proposed and the scope of the research has progressively evolved towards a more specific kind of modelling. These works adopted the constitutive framework of limit analysis to characterise the behaviour of an element under blast loading, which assumes a rigid-perfectly plastic behaviour. While at times restrictive for non-monolithic elements such as sandwich beams (Fleck and Deshpande, 2004) and composites plates (Micallef, 2013), the limit analysis is pertinent to the study of ductile materials without the loss of accuracy.

The theoretical models are, more often than not, cast in dimensionless form to corroborate with the various parameters from the literature and to aid the designer with understanding the phenomenon of blast on the beams without the need to conduct the experimental studies ab initio. Numerical models based on the finite element (FE) method also form a well-established method for obtaining results to be validated with experiments at a later stage. Therefore, theoretical and numerical simulations provide a platform for virtual testing 
of scenarios involving blast loading of different parameters to assess the critical scenarios suitable to be studied further using experimentation.

While most of the available models are concerned with uniform distribution of pressure, an alternative loading profile should be considered in the case of localised blasts to describe the air wave generated by a short-range explosive detonation or a land-mine explosion. Following this rationale, this article seeks to extend the previously mentioned theoretical studies on beams to the cases including localised blast loads through implementation of a modified loading function. Thus, the outline of this article is as follows: following this introduction, a description of localised blast is presented, while the static collapse load is determined in section 'Theoretical Method of analysis'. Considering interactive yield conditions incorporating the effects of bending moment and transverse shear and bending moment and membrane forces, the dynamic behaviour of simply supported and fully clamped beams are ensued, where the influence of bending moment transcends the transverse shear and membrane forces. A theoretical procedure to determine the behaviour of the beam upon the effects of the transverse shear sliding and membrane forces is outlined in sections 'Dynamic plastic collapse of stocky beams' and 'Dynamic plastic response of thin membranes', respectively, followed by influence of the strain-rate sensitivity in the next section. The theoretical analyses are validated against the FE models set up and run using ABAQUS in section 'Finite Element Analyses'. Finally, the concluding remarks of this study are presented in the last section.

\section{Localised blast load}

In most of the works of the literature, the blast load function is a truncated series of multiplicative representation as the product of its spatial (load shape) and temporal (pulse shape) pressure distribution, that is, $P(x, t)=p(x) p^{*}(t)$, where $p^{*}(t)$ is the dimensionless load function representing the temporal pulse shape. The spatial distribution of the blast is commonly assumed to have a profile illustrated in Figure 1 (Bonorchis and Nurick, 2009; Karagiozova et al., 2010; Micallef et al., 2015), representative of a uniform pressure within the central zone of radius $R_{0}$, before exponentially decaying as it spreads away from the origin of the detonation. Thus, the loading function has a symmetric profile about the midspan of the target beam, reducing the domain of study to only one half of the beam span. The temporal distribution of the load depends on the type of explosion, that is, detonation of high explosives or deflagration/deflagration-detonation transition of flammable gas. The type and speed of the wave that propagates through the medium during these explosions would affect blast pressure, constituting different pulse shapes such as rectangular, triangular, exponential or sinusoidal (Fallah and Louca, 2007; Youngdahl, 1971). In this work, however, the temporal distribution of the load is representative of a rectangular pulse as in Figure 2. The specific case of the impulsive load, where the loading time, $\tau$, tends towards 0 , will also be discussed in the following sections.

\section{Yield surface}

The mechanism of deformation in a beam subjected to a centrally localised blast load is the creation of one central or two symmetrical plastic hinges. Provided the elastic energy stored in the system is small compared to the kinetic energy imparted to the structure, and the 


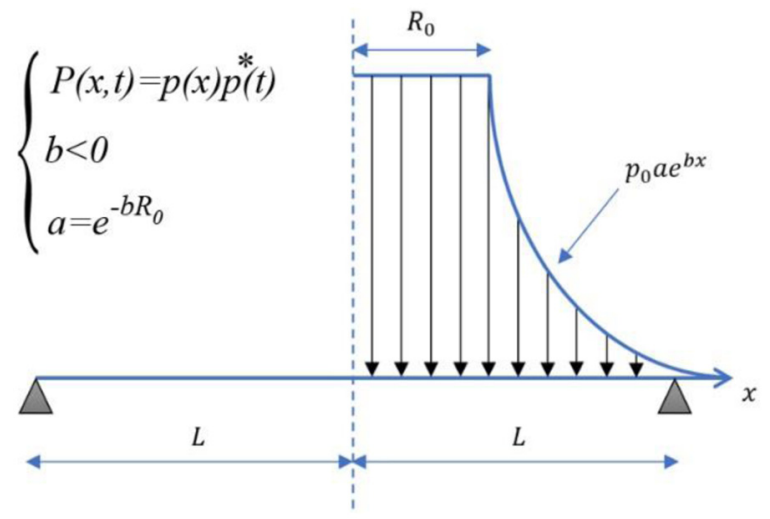

Figure I. Localised blast distribution.

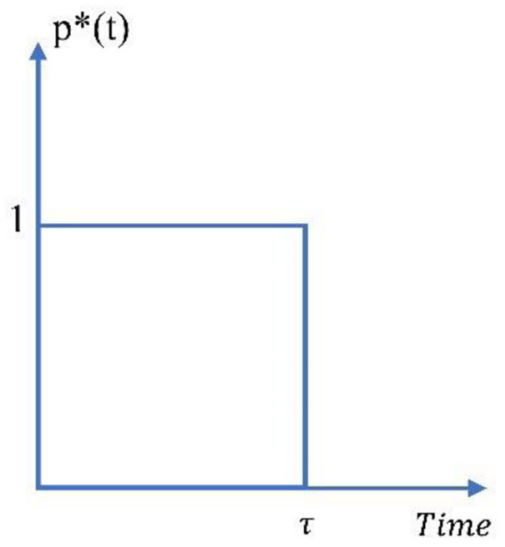

Figure 2. Temporal rectangular pulse shape with load duration $t=\tau$.

duration of the load is smaller than the system's natural period of vibration, the elasticplastic distribution of stresses through the thickness of the cross-section and elastic stress wave propagation may be ignored, the rigid-perfectly plastic behaviour can be assumed and the plastic hinges may be considered as plastic joints (Hopkins and Prager, 1953; Jones, 1990; Jones and Kim, 1997; Ma et al., 2010). This assumption is the cornerstone of the constitutive framework of limit analysis. The number of plastic hinges and the final transverse displacement of the beam depend on the distribution of the pulse load. For a uniform distribution of pressure, only the amplitude of the pulse can influence the nature of the response, while for a localised blast, the deformation of the beam also depends on the load shape.

Hence, it is assumed that the beam is made of a rigid, perfectly-plastic, rate-insensitive material and the plastic flow is controlled by a convex yield surface interactive of the bending moment $M$, shear force $Q$ and membrane forces $N$, creating a three-dimensional yield curve of $M-N-Q$. For brevity in analyses, this full interaction surface is reduced into a 


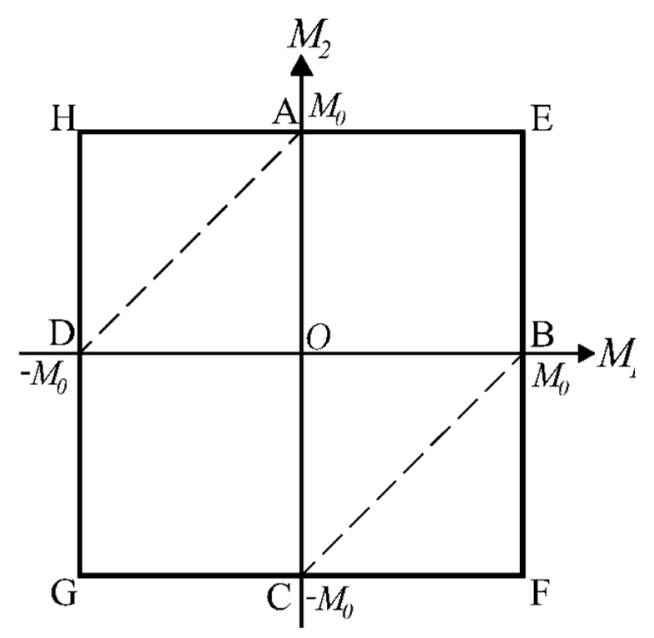

Figure 3. Square yield surface of the beam (EFGH) in bending vs the hexagonal Tresca yield condition ( -$)$ in bending.

two-dimensional (2D) yield curve by ignoring the in-plane deformations which reduces the study to considering yield curve interactive of (1) bending, (2) combined bending and transverse shear and (3) combined bending and membrane forces, as illustrated in Figures 3 to 5, with associated flow rule. The collapse bending moment, $M_{0}$, the plastic collapse transverse shear, $Q_{0}$, and the collapse membrane of the beam, $N_{0}$, are defined in equations (1a) to (1c). According to the normality requirement of Drucker's stability postulate, the state of the strains and the normal to (each) yield surface at each point along the yield path are co-directional, say, in Figure 5, the plastic flow initiates at corner A and moves along path AB (in the case of circumscribing square yield surface, plastic flow is at corner E) until the membrane forces are fully developed $\left(N=N_{0}\right.$ and $\left.M=0\right)$ at corner B.

In the sequel that follows, the mathematical equations are treated by assuming an appropriate statically and kinematically admissible velocity profile in each study.

$$
\begin{aligned}
M_{0} & =\frac{\sigma_{0} \bar{B} H^{2}}{4} \\
Q_{0} & =\frac{\sigma_{0} \bar{B} H}{2} \\
N_{0} & =\sigma_{0} \bar{B} H
\end{aligned}
$$

\section{Theoretical method of analysis}

Many theoretical analyses have been conducted on the response of ductile beams and plates to a wide range of static and dynamic pressure loadings which give rise to plastic material response. The analysis developed in some studies (Jones, 1971, 1990; Jones and Walters, 1983) uses Green's theorem to simplify the general equation of motion for arbitrary shaped 


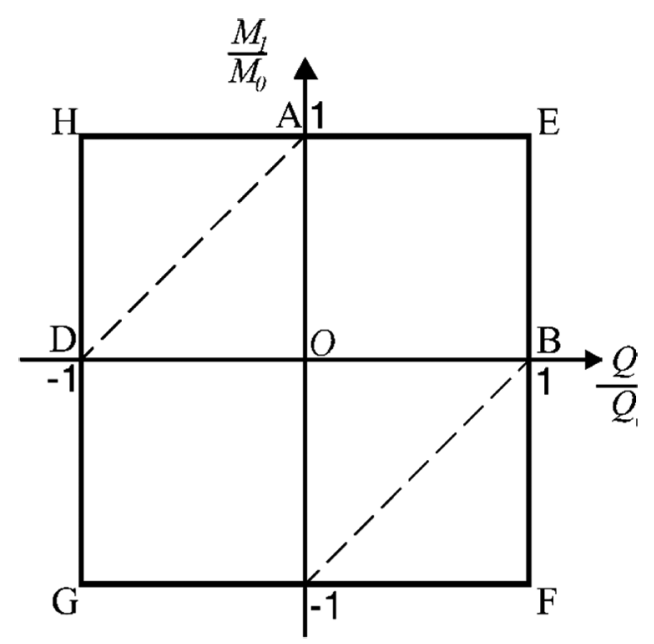

Figure 4. Square yield surface of the beam (EFGH) in bending vs the hexagonal Tresca yield condition ( -$)$ interactive of transverse shear and bending moment.

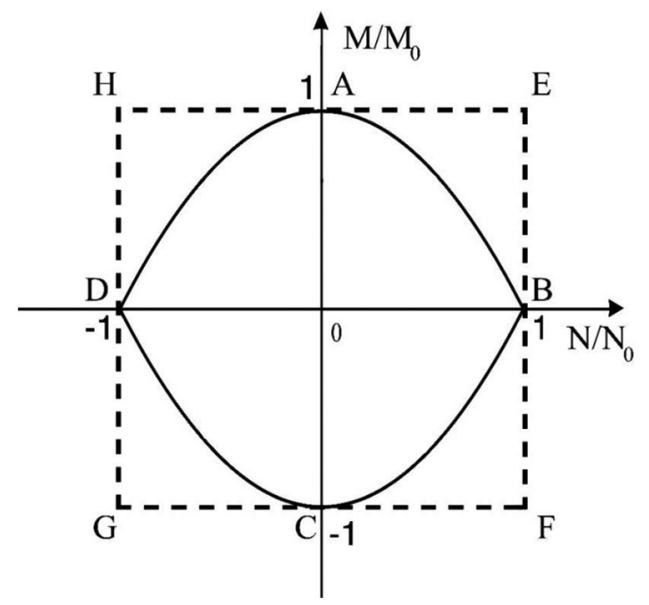

Figure 5. Exact yield surface interactive of membrane and bending (parabola) vs the circumscribing yield surface $(-)$.

plates (and beams as a special case), leading to a set of energy equilibrium equations. Ignoring the in-plane deformations and rotatory inertia effects, the energy equilibrium equation may be expressed as

$$
\int_{\bar{A}}(P(x, t)-\mu \ddot{w}) \dot{w} d \bar{A}=\int_{\bar{A}}(M+N w) \dot{\kappa} d \bar{A}+\sum_{j=1}^{n} \int_{A}(M+N w) \dot{\theta}_{m} d C_{m}+\sum_{u=1}^{v} Q(\dot{w})_{u} d C_{u}
$$


The expression on the left-hand side gives the external work rate over the area $(\bar{A})$ of the element. The first term on the right-hand side expresses the energy dissipation rate in continuous velocity field; the second term on the right-hand side expresses the energy dissipated in $j$ discrete plastic hinges, each having a length of $C_{j}$ and rotational velocity of $\dot{\theta}_{j}=\partial \dot{w} / \partial x_{j}$. The last term on the right-hand side expresses the energy dissipation rate due to transverse shear force at $u$ plastic hinges of length $C_{u}$ and velocity discontinuity of $(\dot{w})_{u}$. Equation (2) ensures the energy conservation in the system.

In this study, a similar procedure is employed to extend the previous theoretical solutions with consideration of the localised blast on ductile inelastic beams. In this work, we shall, as is customary, show the differentiation with respect to time by placing a dot above the term.

\section{Static analysis}

Consider a beam of rectangular cross section $B \times H$ that is made of rigid-perfectly plastic material and pinned at its both ends. The beam is subjected to a lateral pressure load $p(x)$ which is time independent. Tresca' s yield criterion for the beam associated with the plastic collapse of the beam states $|M| \leq M_{0}$, given the maximum bending moment is defined in equation (1a). The global equation of equilibrium for a beam subjected to a pressure $P(x, t)$ reduces to equations (3) and (4), where $m_{b}$ is the linear mass density of the beam

$$
\begin{gathered}
\frac{\partial^{2} M}{\partial x^{2}}+P(x, t)-m_{b} \ddot{w}=0 \\
Q=\frac{\partial M}{\partial x}
\end{gathered}
$$

As the beam is loaded statically, the inertia term of equation (3) is ignored and the incipient plastic collapse (lower bound) is evaluated through direct integration of equilibrium equation (5). Regarding the change of the loading profile at $x=R_{0}$, equation (3) may be treated in two zones, given by

$$
\begin{gathered}
\frac{\partial^{2} M}{\partial x^{2}}+p(x)=0 \\
\frac{d^{2} M}{d x^{2}}= \begin{cases}-p_{0}, & 0 \leq x \leq R_{0} \\
-p_{0} a e^{b x}, & R_{0} \leq x \leq L\end{cases}
\end{gathered}
$$

The expression of bending moment along the beam is obtained through two consecutive spatial integrations of equation (6). The constants of integration $(A-D)$ in equation (7) are then determined by applying the boundary conditions at $M(x=0)=M_{0}, Q(x=0)=0$ and the continuity of the bending moment and shear force at $x=R_{0}$. The constants of integrations are presented in equations (8a) to (8d)

$$
M(x)= \begin{cases}-\frac{p_{c} x^{2}}{2}+A x+B, & 0 \leq x \leq R_{0} \\ -\frac{p_{c} a}{b^{2}} e^{b x}+C x+D, & R_{0} \leq x \leq L\end{cases}
$$




$$
\begin{gathered}
A=0 \\
B=M_{0} \\
C=p_{c}\left(\frac{a}{b} e^{b R_{0}}-R_{0}\right) \\
D=M_{0}+p_{c} \frac{a}{b} e^{b R_{0}}\left(\frac{1}{b}-R_{0}\right)+\frac{p_{c} R_{0}^{2}}{2}
\end{gathered}
$$

Invoking the boundary condition at the end of the beam (i.e. $M(x=L)=0$ ) yields the lower bound static plastic collapse pressure of equation (9), where $\alpha$ is defined in equation (10) as a function of the loading parameters $\left(a, b, R_{0}\right)$

$$
\begin{gathered}
p_{c}=\frac{M_{0}}{\alpha} \\
\alpha=\frac{a}{b^{2}} e^{b L}+\frac{a}{b} e^{b R_{0}}\left(R_{0}-L-\frac{1}{b}\right)+\left(R_{0} L-\frac{R_{0}^{2}}{2}\right)
\end{gathered}
$$

In a similar fashion, the upper bound collapse pressure is determined by equating the external work rate due to imposed load on the beam with its internal energy dissipation rate as in equation (12). Considering an axisymmetric, kinematically admissible velocity profile over the beam span given by equation (11), the rotational velocity $\dot{\theta}=\dot{W} / L$ furnishes the expression of equation (2) into equation (12) when the membrane and transverse shear forces are ignored

$$
\begin{gathered}
\dot{w}=\dot{W}\left(\frac{1-x}{L}\right) \\
2 \dot{\theta} M_{0}=2 \dot{W}\left[\int_{0}^{R_{0}} p_{c}\left(1-\frac{x}{L}\right) d x+\int_{R_{0}}^{L} p_{c} a e^{b x}\left(1-\frac{x}{L}\right) d x\right]
\end{gathered}
$$

The critical value for the static collapse pressure would thus be given as

$$
p_{c}=\frac{M_{0}}{\beta}, \quad \text { with } \beta=\alpha
$$

Clearly, the upper and lower bound collapse pressures are identical and the static plastic collapse of equation (13) is, therefore, exact. Moreover, it is mathematically evident that in both cases, as $a \rightarrow 1$ and $b \rightarrow 0$, expression in equation (9) simplifies to $p_{c}=2 M_{0} / L^{2}$ which is the case of uniform pressure. Similarly, with $a \rightarrow 0$ and $b \rightarrow-\infty, P_{c}=2 M_{0} / L^{2}$ that is identical to the case of a point load $\left(P_{c}\right)$.

While it is physically reasonable to assume the conical shape in equation (11) for the velocity profile, it is mathematically evident that the expressions studied here (including the velocity profile) converge to the expressions for both concentrated force with $b \rightarrow-\infty$ and for uniform load with $b \rightarrow 0$, therefore is not unreasonable to assume the same velocity profile for $0<b<\infty$, that is, the localised blast case. 


\section{Dynamic analyses}

Dynamic analyses are carried out by including the inertia term of equation (3) in the analyses. We shall denote the dynamic load factor by $\eta$ (equation (14)) defined as the ratio of the dynamic pressure load and the critical static collapse pressure of equation (13). The nature of the dynamic plastic behaviour, that is, the number of plastic hinges, their positions and velocities, is determined by the value of this factor; in other words, while for the range of $0 \leq \eta \leq 1$, no deformation would occur, and the kinematic relations of bending moment for $\eta>1$ give rise to two distinct cases separated by a critical value of dynamic load factor (to be obtained in equation (22)):

(1) Case 1: $\eta \leq \eta_{\text {crit }}$ in which the velocity profile takes the shape of Figure 6(a).

(2) Case 2: $\eta \geq \eta_{\text {crit }}$ in which the velocity profile is represented as in Figure 6(b) with a time-dependent plastic hinge position. The plastic hinges move inward and the profile ultimately develops into the profile of Figure 6(a)

$$
\eta=\frac{p_{0}}{p_{c}}
$$

Case $I: \eta \leq \eta_{\text {crit }}$. The velocity profile may be assumed as in equation (11), with only one static plastic hinge forming at either side of the beam midspan. The beam response is governed by two distinctive phases, that is, $0 \leq t \leq \tau$ and $\tau \leq t \leq T$, where $\tau$ and $T$ are the durations of the load and the time of permanent deformation, respectively.

In the first phase of motion, a similar procedure as in the previous section is utilised, but with the inertia term included, and two consecutive integrations of equation (3) leads to equation (15), with the constants $A-D$ identical to those previously defined in equations (8a) to $(8 d)$

$$
M_{x}= \begin{cases}-\frac{p_{0} x^{2}}{2}+m_{b}\left(\frac{x^{2}}{2}-\frac{x^{3}}{6 L}\right) \ddot{W}+A x+B, & 0 \leq x \leq R_{0} \\ -\frac{p_{0} a}{b^{2}} e^{b x}+m\left(\frac{x^{2}}{2}-\frac{x^{3}}{6 L}\right) \ddot{W}+C x+D, & R_{0} \leq x \leq L\end{cases}
$$
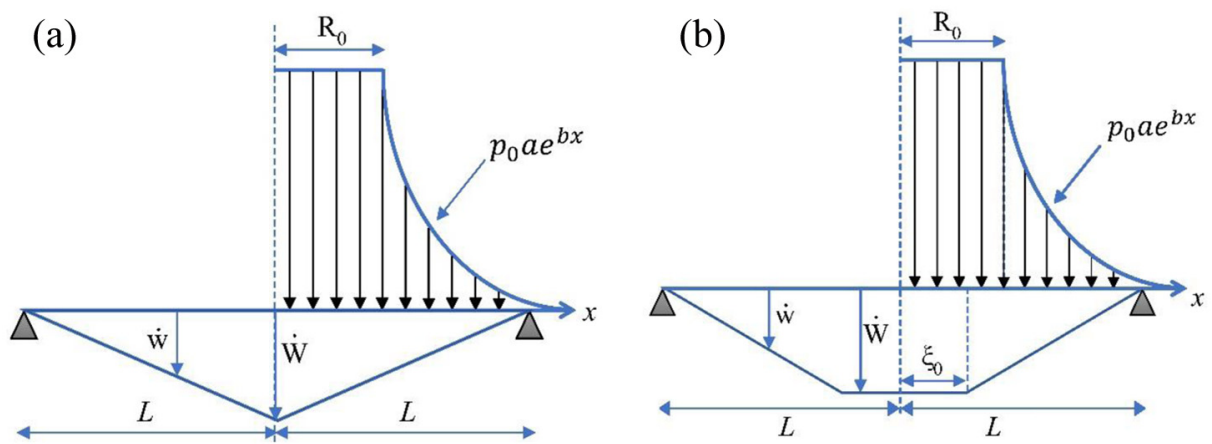

Figure 6. (a) Velocity profile of the beam in case I; (b) velocity profile with travelling plastic hinges. 
The condition of simply supported boundaries $(M(x=L)=0)$ gives rise to the transverse acceleration of the beam as in equation (16). At time $t=0$, the deformation is uniformly zero, thus the displacement of the beam, that is, equation (17), can be evaluated by time integration of the inertia term

$$
\begin{aligned}
\ddot{W} & =\frac{3 M_{0}}{m_{b} L^{2}}(\eta-1) \\
W_{1} & =\frac{3(\eta-1)}{2 m_{b} L^{2}} M_{0} \tau^{2}
\end{aligned}
$$

In the second phase of motion, the pressure load vanishes. However, the beam contains some residual kinetic energy, which must be dissipated before the motion ceases. Hence, the transverse displacement continues to increase till the velocity of the beam vanishes. With the inertia term reducing to equation (18), followed by two time integrations, the transverse displacement in this phase is achieved. The constants of integration are obtained by ensuring the kinematic admissibility of transverse displacement and transverse velocity at $t=\tau$. Subsequently, the motion terminates at time $T>\tau$ when the transverse velocity vanishes. The permanent midspan deformation corresponding to this time is determined by equation (19)

$$
\begin{gathered}
\ddot{W}=\frac{-3 M_{0}}{m_{b} L^{2}} \\
W_{f}=3 \eta(\eta-1)\left(\frac{M_{0} \tau^{2}}{2 m_{b} L^{2}}\right)
\end{gathered}
$$

It has not yet been established whether the analytical solution violates the yield condition at any phase of motion. Indeed, for the beam system to be statically admissible, the requirements of equations (20) and (21) must be satisfied. Clearly, the expression of bending moment at midspan in equation (15) satisfies the requirement of equation (20). The requirement of equation (21) leads to an expression for $\eta_{\text {crit }}$ as a function of the loading parameters presented in equation (22). Take $a \rightarrow 1, b \rightarrow 0$ and $R_{0} \rightarrow R$, for example, the critical value of dynamic load factor, $\eta_{\text {crit }} \rightarrow 3$, which is identical to the condition for the uniform load derived in Jones (1990)

$$
\begin{gathered}
\left.\frac{d M}{d x}\right|_{x=0}=0 \\
\left.\frac{d^{2} M}{d x^{2}}\right|_{x=0} \leq 0 \\
\eta \leq \frac{3}{3-\left(L^{2} / \alpha\right)}=\eta_{\text {crit }}
\end{gathered}
$$

Case 2: $\eta \geq \eta_{\text {crit }}$. When the magnitude of the dynamic load factor exceeds the limiting value $\left(\eta_{\text {crit }}\right)$, the admissibility condition in equation (21) is violated, dictating a required modification to the velocity profile of the previous section. It is assumed that the velocity profile at the onset of motion in this case is governed by that of Figure 6(b), with two symmetric 
incipient plastic hinges formed at either side of the beam, each having a distance $\xi_{0}$ from the midspan. Hence, the central region $\left[-\xi_{0} ; \xi_{0}\right]$ is controlled by the continuous plasticity where the plastic bending moment acquires it maximum $M_{0}$ while the transverse shear $Q$ vanishes. Throughout the motion, however, the size of this region tends to reduce because a timedependent plastic hinge $\xi(t)$, developed in the central zone, travels inwards, that is, towards the centre of the beam. The central incipient plastic hinges occur to ensure the rate of strain energy equates the work done at every instant of time. With this rationale in mind, three distinguished phases are outlined in this case, as follows.

First phase of motion $(0 \leq t \leq \tau)$. The loading conditions, mentioned in the previous part, are maintained in this case. However, the velocity profile has been modified due to the appearance of the two plastic hinges at $x=-\xi_{0}$ and $x=\xi_{0}$. Although in the initial analysis we assumed $R_{0} \leq \xi_{0}$, further calculations proved that this assumption must be adopted for the beam system in order to avoid a contradiction. Hence, the velocity profile that has been considered for this section is the one displayed in equation (23) with $\xi_{0} \leq R_{0}$

$$
\dot{w}= \begin{cases}\dot{W}_{1}, & 0 \leq x \leq \xi_{0} \\ \dot{W}_{1} \frac{L-x}{L-\xi_{0}}, & \xi_{0} \leq x \leq R_{0} \\ \dot{W}_{1} \frac{L-x}{L-\xi_{0}}, & R_{0} \leq x \leq L\end{cases}
$$

Now, the integration of equation (3) gives a new distribution of $M(x)$ along the beam as in equation (24)

$$
M(x)= \begin{cases}\left(-p_{0}+m_{b} \ddot{W}_{1}\right) \frac{x^{2}}{2}+A x+B, & 0 \leq x \leq \xi_{0} \\ -\frac{p_{0} x^{2}}{2}+\frac{m_{b} \ddot{W}_{1}}{L-\xi_{0}}\left(\frac{x^{2}}{2}-\frac{x^{3}}{6 L}\right)+C x+D, & \xi_{0} \leq x \leq R_{0} \\ -\frac{p_{0} a}{b^{2}} e^{b x}+\frac{m_{b} \ddot{W}_{1}}{L-\xi_{0}}\left(\frac{x^{2}}{2}-\frac{x^{3}}{6 L}\right)+E x+F, & R_{0} \leq x \leq L\end{cases}
$$

The condition of $M=M_{0}$ in $\left[0, \xi_{0}\right]$ requires that $m_{b} \ddot{W}-p_{0}=0$, thus

$$
W_{1}=\frac{p_{0} \tau^{2}}{2 m_{b}}
$$

where $\dot{W}_{1}(t=0)=W(t=0)=0$. Enforcing the boundary conditions at $x=0$ and the continuity of both the shear force $Q$ and the bending moment $M$ at $x=\xi_{0}$ and $R_{0}$, respectively, we obtain the integration constants $(A-F)$ as

$$
\begin{gathered}
\left\{\begin{array}{c}
A=0 \\
B=M_{0}
\end{array}\right. \\
\left\{\begin{array}{c}
C=p_{0}\left(\frac{a}{b} e^{b R_{0}}-R_{0}\right) \\
D=M_{0}+p_{0} \frac{a}{b} e^{b R_{0}}\left(\frac{1}{b}-R_{0}\right)+\frac{p_{0} R_{0}^{2}}{2}
\end{array}\right.
\end{gathered}
$$




$$
\left\{\begin{array}{l}
E=p_{0}\left[\frac{a}{b} e^{b R_{0}}-R_{0}-\frac{\xi_{0}^{2}}{2\left(L-\xi_{0}\right)}\right] \\
D=M_{0}+\frac{p_{0} a}{b} e^{b R_{0}}\left(\frac{1}{b}-R_{0}\right)+\frac{p_{0}}{L-\xi_{0}}\left[\frac{R_{0}^{2} L}{2}-\frac{R_{0}^{2} \xi_{0}}{2}+\frac{\xi_{0}^{3}}{6}\right]
\end{array}\right.
$$

Since the bending moment vanishes at the tip of the beam, $M(x=L)=0$, the initial position of the plastic hinges $\xi_{0}$ is established as a function of $\eta$ and $\alpha$ (and vice versa) in equation (28). Clearly, when $\xi_{0}$ approaches zero (almost no travelling hinges), $\eta$ tends towards its value $\eta_{\text {crit }}$ derived in equation (22)

$$
\frac{1}{\eta}=1-\frac{\left[\frac{L^{3}}{3}+\frac{\xi_{0}^{3}}{6}-\frac{L \xi_{0}^{2}}{2}\right]}{\alpha\left(L-\xi_{0}\right)}
$$

Second phase of motion $\left(\tau \leq t \leq T_{2}\right)$. This phase of motion occurs as the pulse load vanishes at $t=\tau$ and the incipient plastic hinges replace with time-dependent plastic hinges $\xi(t)$ which now travel along the length towards the centre of the beam. We write the new moment distribution as

$$
\frac{d^{2} M}{d x^{2}}=\left\{\begin{array}{lc}
m_{b} \ddot{W}_{2}, & 0 \leq x \leq \xi \\
m_{b} \ddot{W}_{2} \frac{L-x}{L-\xi}+m_{b} \ddot{W}_{2} \dot{\xi} \frac{L-x}{(L-\xi)^{2}}, & \xi \leq x \leq L
\end{array}\right.
$$

This equation may be integrated on the $[\xi L]$ interval to yield the expression for $M(x)$ as in equation (30), where the integration constants in equation (31) are obtained due to $Q(x=\xi)=0$ and $M(x=L)=0$

$$
\begin{aligned}
M(x)= & \frac{p_{0} \tau \dot{\xi}}{(L-\xi)^{2}}\left(\frac{L x^{2}}{2}-\frac{x^{3}}{6 L}\right)+C x+D \\
& \left\{\begin{array}{l}
C=-\frac{p_{0} \tau \xi(L-\xi / 2) \dot{\xi}}{(L-\xi)^{2}} \\
D=-\frac{p_{0} \tau L^{3} \dot{\xi}}{3(L-\xi)^{2}}-C L
\end{array}\right.
\end{aligned}
$$

Due to the continuity of the bending moment at the centre, $M(x=\xi)=M_{0}$ in equation (30), the expression of equation (32) can be obtained. This expression may be integrated as in equation (33) to determine the position of the plastic hinges $\xi(t)$ in equation (34)

$$
\begin{gathered}
M_{0}=-\frac{p_{0} \tau}{3}(L-\xi) \dot{\xi} \\
\left\{\int_{\xi_{0}}^{\xi}(L-\xi) d \xi=-\int_{\tau}^{t} \frac{3 M_{0}}{p_{0} \tau d t}\right.
\end{gathered}
$$




$$
\begin{gathered}
\frac{\xi^{2}}{2}-L \xi+L \xi_{0}-\frac{\xi_{0}^{2}}{2}=\frac{3 M_{0}(t-\tau)}{p_{0} \tau} \\
L \xi_{0}-\frac{\xi_{0}^{2}}{2}=\frac{3 M_{0}}{p_{0} \tau}\left(T_{2}-\tau\right)
\end{gathered}
$$

However, the requirement $M(x)=M_{0}$ in the interval $x \in\left[\begin{array}{ll}0 & \xi\end{array}\right]$ has yet to be verified. This condition dictates $m \ddot{W}=0$, deduced from equations (29) and (5), since the loading is absent and the central zone moves with a constant velocity. Invoking the kinematic admissibility at $t=\tau, \dot{W}_{2}(t=\tau)=\dot{W}_{1}(t=\tau)$ and $W_{2}(t=\tau)=W_{1}(t=\tau)$, we obtain the subsequent description of the transverse displacement at the end of the second phase as in equation (36). This phase terminates when these hinges reach the midspan of the beam (i.e. when $\xi=0)$ at $t=T_{2}$, expressed by equation (35). Thus, the end time of this phase can be determined as in equation (37), by substituting the expression of $\xi_{0}$ in equation (28) into equation (35). The expressions of plastic hinge and its velocity are presented in Figure 7

$$
\begin{gathered}
W_{2}=\frac{p_{0} \tau}{m_{b}}\left(t-\frac{\tau}{2}\right) \\
T_{2}=\left(\frac{3 \alpha}{L^{2}}-1\right) \frac{p_{0} \tau L^{2}}{3 M_{0}}
\end{gathered}
$$

Clearly, if $a \rightarrow 1, b \rightarrow 0$ and $R_{0} \rightarrow L$, then $T_{2} \rightarrow p_{0} \tau L^{2} / 6 M_{0}$, which is the case of the uniform pressure load studied in the literature (Jones, 1990).

Third phase of motion $\left(T_{2} \leq t \leq T_{f}\right)$. The plastic hinge line in the central zone is now vanished and the beam takes the profile shape in Figure 6(a). The fundamental difference between this

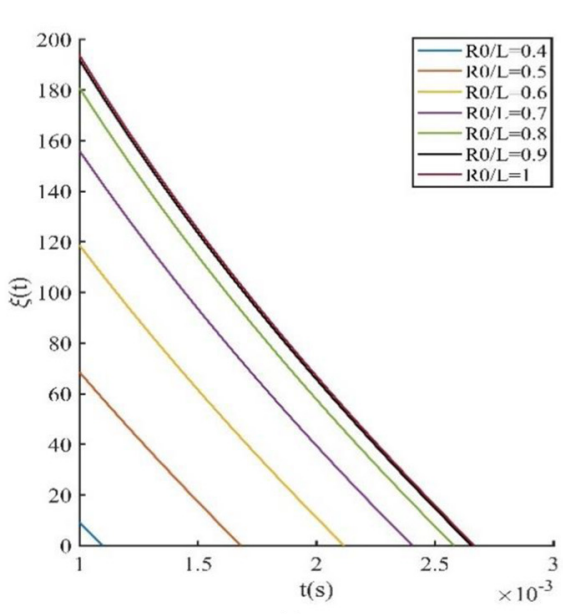

(a)

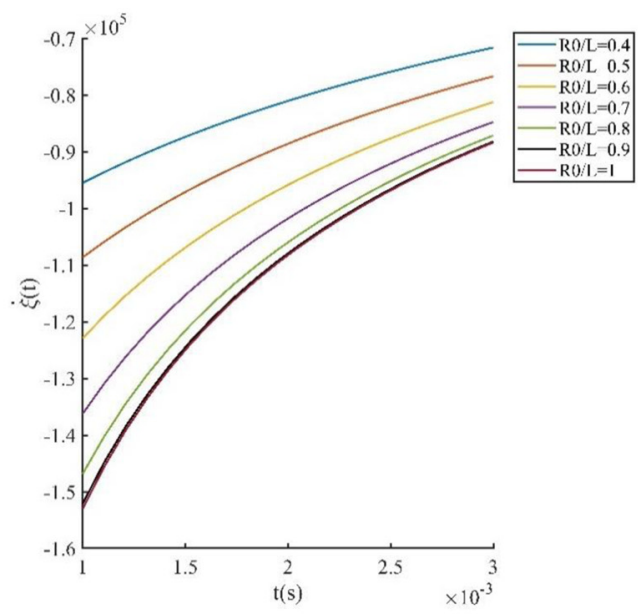

(b)

Figure 7. (a) Variation of the travelling hinge (equation (34)) and (b) its velocity over time (equation (32); for reference to the interpretation of colour in this figure legend, refer to the online version). 
case and the case of a single central plastic hinge is that, despite similar final displacement profiles, in this case, there is a plasticised zone which is absent in the latter case. However, as in the previous case, the reserved kinetic energy must be dissipated before the beam finally comes to rest. Thus, the motion continues but with the inertia of the beam identical to the previous case as in equation (18), that is, $\ddot{W}_{3}=-3 M_{0} / m_{b} L^{2}$, which is integrated to yield the transverse velocity and displacement in equations (38) and (39) when the kinematic admissibility at $t=T_{2}$ is considered

$$
\begin{gathered}
\dot{W}_{3}=-\frac{3 M_{0}}{m_{b} L^{2}} t+\frac{3 p_{0} \tau \alpha}{m_{b} L^{2}} \\
W_{3}=-\frac{3 M_{0}}{2 m_{b} L^{2}} t^{2}+\frac{3 p_{0} \tau \alpha}{m_{b} L^{2}} t-\frac{p_{0} \tau^{2}}{m_{b}}-\frac{p_{0}^{2} \tau^{2} L^{2}}{6 m_{b} M_{0}}\left(\frac{3 \alpha}{L^{2}}-1\right)^{2}
\end{gathered}
$$

Finally, when $\dot{W}_{3}=0$, all the energy is expended in plastic work and the beam comes to rest. This gives $T_{f}=\eta \tau$ and the permanent transverse displacement as in equation (40)

$$
W_{f}=\frac{p_{0}^{2} \tau^{2} L^{2}}{m_{b} M_{0}}\left(\frac{\alpha}{L^{2}}-\frac{1}{6}\right)-\frac{p_{0} \tau^{2}}{2 m_{b}}
$$

The foregoing analysis for the simply supported beam can be extended to the fully clamped beams. With the boundary conditions at the tip of the beam $M(x=L)=-M_{0}$ in mind, the static collapse pressure is identified as

$$
\overline{p_{c}}=\frac{2 M_{0}}{\alpha}
$$

Considering this modification, the expressions for the simply supported beam can be utilised for the fully clamped case, by only changing $M_{0}$ into $2 M_{0}$ and $\eta$ with $\bar{\eta}$, with $\bar{\eta}$ being defined as $\bar{\eta}=p_{0} / \overline{p_{c}}$. Using this rationale, the expressions of the maximum final transverse displacement would be modified to

$$
\begin{gathered}
\bar{\eta} \leq \overline{\eta_{\text {crit }}} \rightarrow W_{f}=3 \bar{\eta}(\bar{\eta}-1) M_{0} \tau^{2} / m_{b} L^{2} \\
\bar{\eta}>\overline{\eta_{\text {crit }}} \rightarrow W_{f}=\frac{p_{0}^{2} \tau^{2} L^{2}}{2 m_{b} M_{0}}\left(\frac{\alpha}{L^{2}}-\frac{1}{6}\right)-\frac{p_{0} \tau^{2}}{2 m_{b}}
\end{gathered}
$$

As expected, it can be easily verified that the statement $W_{f(\text { fully clamped })}<W_{f(\text { simply supported })}$ holds in each loading case $\left(\eta<\eta_{\text {crit }}\right.$, or $\left.\eta>\eta_{\text {crit }}\right)$.

\section{Dynamic plastic collapse of stocky beams}

Consider now the beam made of ductile material and rectangular cross section with depth $H$ and half-breadth $L$. It has been shown that as the thickness-to-length ratio of the beam increases, the contribution of the transverse shear strain becomes significant, causing the transverse shear sliding at the supports to influence the dynamic response. The yield surface thus consists of interaction between the plastic collapse bending moment and the plastic transverse shear force as per Figure 4, which would be given as in equation (1a) and equation (1b). 
Using the expressions of bending moment from the static analysis in previous section, the static transverse shear force $Q_{s}$ at the tip of the beam is evaluated by equation (43). The quotient $Q_{S} / Q_{0}$, as in equation (44), could be related to aspect ratio of the beam as a direct measure of the beam's geometry (i.e. ratio $H / L$ ). Provided this ratio is infinitesimal (which is valid in most beam profiles), the static shear sliding effects do not considerably influence the response of the beam (see equation (45)).

In a similar fashion, the shear sliding effects may be evaluated for dynamic loads, as expressed in equations (46) to (49). Clearly, the dynamic shear sliding will be significant as the load approaches impulsive, that is, when $\eta \rightarrow \infty$ and $\tau \rightarrow 0$. This is expected because when the pulse duration decreases, the beam loses its flexibility in bending, forcing the energy to be absorbed through shear

$$
\begin{gathered}
Q_{s}(x=L)=\frac{M_{0}}{\alpha}\left(\frac{1}{b}-\frac{a e^{b L}}{b}-R_{0}\right) \\
\frac{Q_{s}}{Q_{0}}=\frac{H}{2 \alpha}\left(\frac{1}{b}-\frac{a e^{b L}}{b}-R_{0}\right) \overrightarrow{\text { uniform }}-\frac{H}{L} \\
\left|Q_{s} / Q_{0}\right| \ll 1 \quad \text { when } H / L<1 \\
\frac{Q_{d}}{Q_{0}}=\left[\frac{a}{b}\left(e^{b R_{0}}-e^{b L}\right)-R_{0}+\frac{L+\xi_{0}}{2}\right] p_{0} \\
\frac{Q_{d}}{Q_{0}} \underset{\text { uniform }-\frac{H}{2 L} \eta\left(1-\frac{\xi_{0}}{L}\right)=-\frac{H}{2 L} \sqrt{3 \eta}}{\left.\mid \frac{Q_{0}}{2 L}\right)-R_{0}+\frac{p_{0} H \eta}{2 \alpha}} \\
\left|\frac{Q_{d}}{Q_{0}}\right| \rightarrow \text { if } \eta \rightarrow \infty
\end{gathered}
$$

In the case of impulsive loading, the beam acquires an instantaneous velocity $V_{0}$, which must satisfy the momentum conservation of equation (50). The impulsive velocity due to the uniform loading, given by $V_{1}=p_{0} \tau / m$ as in equation (51), may be furnished in terms of the impulsive velocity for the localised load $V_{0}$ in equation (51). Subsequently, the theoretical procedure for the beams subjected to localised blast is identical to the uniform load except the new impulsive velocity $V_{1}$, which could be used in lieu of the uniform impulsive velocity. In other words, the description of the beam's transverse displacement for the localised loads, as given in Table 1 , might be merely obtained by replacing $V_{0}$ by $V_{1}$ into the expressions of the uniform load case in Jones (1990)

$$
\begin{gathered}
\int_{0}^{\tau} F d t=\int_{0}^{\tau} d\left(m_{b} v\right) \\
V_{0}=\frac{p_{0} \tau}{m_{b} L b}\left(a e^{b L}-a e^{b R_{0}}+b R_{0}\right) \Leftrightarrow\left\{\begin{array}{l}
V_{1}=\frac{p_{0} \tau}{m_{b}} \\
V_{1}=V_{0} \frac{b L}{\left(a e^{b L}-a e^{b R_{0}}+b R_{0}\right)}
\end{array}\right.
\end{gathered}
$$


Table I. Transverse displacement for different values of $\nu$.

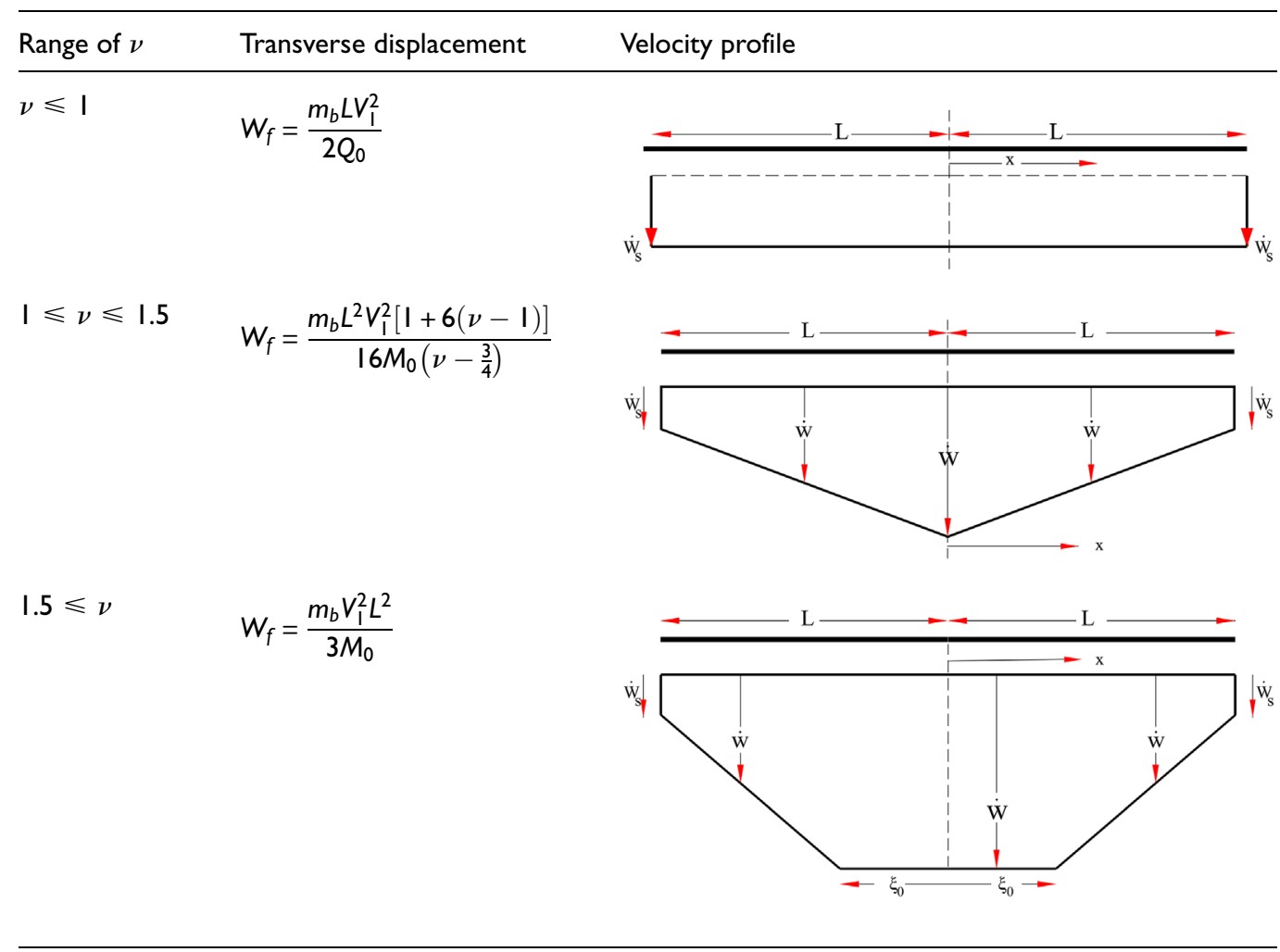

Introducing a new dimensionless number $\nu=Q_{0} L / 2 M_{0}$, this ratio characterises the proportions of shear and bending resistance of the beam and is only a measure of beams' geometry for prismatic sections since it reduces to $\nu=L / H$. Depending on the range of $\nu$, three cases can be distinguished as follows, where the permanent deflection in each case is outlined in Table 1. These cases are as follows

- $\quad \nu \leq 1$, where the shear sliding effect prevails;

- $1 \leq \nu \leq 1.5$, where there is a competition between the bending and shear effects;

- $1.5 \leq \nu$, where the main resistance of the beam is provided through bending effects.

\section{Dynamic plastic response of thin membranes}

It is widely accepted that, when the deflection of the beam increases to the order of its thickness, the membrane forces will be induced, and these forces resist the structural deformation and strengthen the beam's stiffness significantly. The appearance of the membrane forces alters the dynamic equilibrium, and the yield condition in bending, $\left(M(x=0)=M_{0}\right)$, discussed previously, remains valid but only at the onset of motion. Subsequently, by referring to the yield condition for simply supported beams in finite displacements (Jones, 1971; Yu 
and Chen, 1992), it may be assumed that the yield locus is controlled by the interaction of bending moment and membrane in the form of a convex surface as

$$
\left|\frac{M}{M_{0}}\right|+\left(\frac{N}{N_{0}}\right)^{2}=1
$$

The equilibrium condition can be written as equation (53), which may be solved by enforcing the initial and boundary conditions. The procedure to derive the static plastic pressure is outlined in the sequel as equations (54) to (57)

$$
\begin{gathered}
\frac{d Q}{d x}+\frac{d(N d w / d x)}{d x}+p=0 \\
\left\{\begin{array}{l}
\frac{d N}{d x}=0 \\
\frac{d^{2} w}{d x^{2}}=0
\end{array} \Rightarrow M(x)=\left\{\begin{array}{rr}
-\frac{p x^{2}}{2}+A x+B \quad 0 \leq r \leq R_{0} \\
-\frac{p a}{b^{2}} e^{b x}+C x+D \quad R_{0} \leq r \leq L
\end{array}\right.\right. \\
\left\{\begin{array}{l}
A=-\frac{N W}{L} \\
B=p \alpha-N W \\
C=p\left[\frac{a}{b} e^{b R_{0}}-R_{0}\right]-\frac{N W}{L} \\
D=0 \\
M(x=0)=p \alpha-N W \Rightarrow\left[\frac{p_{0} \alpha}{M_{0}}+\frac{N W}{M_{0}}\right]+\left(\frac{N}{N_{0}}\right)^{2}=1
\end{array}\right. \\
\frac{p}{p_{c}}=1+\frac{4 W^{2}}{H^{2}}
\end{gathered}
$$

The foregoing static analysis is extended to consider the transverse inertia term in the dynamic equilibrium equation. It should be mentioned that, when the membrane forces enter the equation of motion, the exact theoretical solution to predict the response of the beam is intrinsically difficult to obtain even for simple problems and some simplifications are necessary to derive the state of stress at any yield points. By referring to the theoretical analyses of Jones $(1971,2014)$ for the inelastic membrane, the energy equilibrium in equation (2) can be written in a form which is resulted when ignoring the transverse shear, in-plane and rotatory inertia

$$
\int_{A}\{p(x, t)-\mu \ddot{w}\} \dot{w} d A=\sum_{j=1}^{n} \int_{l_{m}}[M+N w] \dot{\theta}_{j} d l_{j}
$$

where $\mu$ is the mass per surface area and other parameters as defined previously. The righthand side of the equation is the energy dissipated in $n$ distinct plastic hinges, each of length $l_{m}$ and characteristic angular velocity $\dot{\theta}=(\partial \dot{w} / d x)_{j}$. Equation (58) ensures the external work rate equals the internal energy dissipation rate. 
It may be assumed that plastic flow is controlled by the square yield curve which circumscribes the exact curve given in Figure 5. Thus, $M=M_{0}$ and $N=N_{0}$ as given in equations (1a) and (1c), respectively. Considering an axisymmetric, kinematically admissible deformation field given by $\dot{w}=\dot{W}(1-x / L)$, the right-hand side of equation (58) simplifies to $2 \dot{\theta} B\left(M_{0}+N_{0} W_{1}\right)$, giving rise to equations (59) and (60), where $W_{1}$ is the central transverse deflection in the first phase of motion (i.e. $0<t \leq \tau$ ). Equation (60) is a second-order, nonhomogeneous linear ordinary differential equation. This equation has a general solution as in equation (61) when satisfying the initial conditions at the onset of motion (i.e. $W_{1}=0$ and $\dot{W}=0$ at $t=0$ ). The subsequent expressions are obtained by adopting a similar procedure to the case of infinitesimal deformations. Hence, using the kinematic admissibility at $t=\tau$, the subsequent deformation of the beam for $\tau \leq t \leq T$ is formulated as in equation (63), where $T$ is the time of the completion of permanent deformation (when the beam comes to rest). $a_{1}=m_{b} L^{2} / 3 M_{0}$ and $a_{2}=4 / H$ are the coefficients of the transverse acceleration and transverse displacements, respectively, derived based on equation (60), while $a_{3}^{2}=a_{2} / a_{1}$ given in equation (62)

$$
\begin{gathered}
\frac{m_{b} L^{2}}{3 M_{0}} \ddot{W}_{1}+\frac{4}{H} W_{1}=\frac{p_{0} \alpha}{M_{0}}-1 \\
a_{1} \ddot{W}_{1}+a_{2} W_{1}=\eta-1 \\
W_{1}=\frac{(1-\eta)\left[\cos \left(a_{3} t\right)-1\right]}{a_{2}} \\
W_{2}=\frac{a_{3}^{2}=\frac{12 M_{0}}{m_{b} L^{2} H}}{\eta_{\sin \left(a_{3} \tau\right) \sin \left(a_{3} T\right)+(\eta-1)\left[\cos \left(a_{3} \tau\right)-1\right] \cos \left(a_{3} t\right)+\cos a_{3} \tau \cos a_{3} t-1}^{a_{2}}} \\
\frac{W_{f}}{H}=\frac{\left[(1+2 \eta)(\eta-1)\left(\cos \left(a_{3} \tau\right)-1\right)\right]^{\frac{1}{2}}-1}{4}
\end{gathered}
$$

Equation (64) gives the dimensionless final displacement of the centre of the beam. In the case of an impulsive load, $\eta$ becomes large and $\tau$ becomes vanishingly small (infinitesimal), thus $\eta-1 \sim \eta$ and $\cos \left(a_{3} \tau\right)-1 \sim a_{3}^{2} \tau^{2} / 2$. The permanent transverse deflection, given as equation (65), may be cast in its dimensionless form in equation (67), where the dimensionless kinetic energy $\lambda$ is defined by equation (66). Clearly, in the case of uniform loads, $R_{0} \rightarrow L$ and the expression in equation (67) simplifies to that of equation (68) when $\lambda=m_{b} V_{0}^{2} L^{2} / M_{0} H$

$$
\begin{gathered}
\frac{W_{f}}{H}=\frac{\left(1+\eta^{2} a_{3}^{2} \tau^{2}\right)^{\frac{1}{2}}}{4}-\frac{1}{4} \\
\lambda=\frac{m_{b} V_{1}^{2} L^{2}}{M_{0} H} \frac{4 \alpha^{2}}{L^{4}} \\
\frac{W_{f}}{H}=\frac{\left[\left\{1+\frac{3 m_{b} V_{1}^{2} L^{2}}{M_{0} H} \frac{4 \alpha^{2}}{L^{4}}\right\}^{\frac{1}{2}}-1\right]}{4}
\end{gathered}
$$




$$
\frac{W_{f}}{H}=\frac{\left[\{1+3 \lambda\}^{\frac{1}{2}}-1\right]}{4}
$$

\section{Strain-rate dependence}

In this section, a brief discussion on the influence of strain-rate sensitivity of the material is presented. Although it has been argued that the influence of the strain-rate sensitivity in some ductile materials, such as mild steel and aluminium, is significant even for low-impact velocities (Jones, 2010), few works are published on the strain-rate sensitivity of the materials as the equations are notoriously difficult to solve analytically.

Symonds and Jones (1972) investigated the large displacements of the impulsively loaded beams. They also studied the influence of the material strain-rate sensitivity (visco-plasticity) in theoretical analyses by incorporating the dynamic flow stress $\sigma_{0}^{\prime}$ known as CowperSymonds equation as given below

$$
n=\frac{\sigma_{0}^{\prime}}{\sigma_{0}}=1+\left(\frac{\dot{\varepsilon}}{D_{1}}\right)^{\frac{1}{q}}
$$

This is a phenomenological material constitutive model, thus the parameters must be determined from the experiments. Commonly, the exponent $q=5$ and the base strain rate $D_{1}=40.4 s^{-1}$ for mild steel give good estimates of the dynamic stress. This highly nonlinear equation gives a crude estimate of the plastic flow, because the strain rate varies both spatially and temporally during the deformation of the structure. However, Perrone and Bhadra (1979) showed that in the analyses of large deflections of the beams, the maximum membrane strain rate is reached when $1 / 2$ of the kinetic energy has been dissipated, that is, when the velocity of the beam reduces to $V_{1} / \sqrt{2}$. This would correspond to the time when the deflection has reached $2 / 3$ of its permanent value. Hence, by extending the expression of the permanent displacement of the beam in large displacement theory as equation (67), the dimensionless permanent displacement ratio $W_{f} / H$ can be transformed into equation (70), where the strain-rate coefficient $n$ is defined as equation (71). At this stage, this is an iterative formula, since $n$ is a function of $W_{f}$. Therefore, by taking $\dot{\varepsilon}=\dot{W} W / L^{2}$ and with the knowledge that $\dot{\varepsilon}$ reaches maximum strain rate at $\dot{W}=V_{1} / \sqrt{2}$, the large displacement assumption must be considered $\left(H \ll W_{f}\right)$, to transform the original expression of the deflection into equation (72) which casts the expression of $n$ into the form of equation (73)

$$
\begin{aligned}
\frac{W_{f}}{H} & =\frac{\left[\left(1+\frac{48 \rho V_{1}^{2}}{n \sigma_{0} H^{2}} \frac{\alpha^{2}}{L^{2}}\right)^{\frac{1}{2}}-1\right]}{4} \\
n & =1+\left[\frac{2 V_{1} W_{f}}{3 \sqrt{2} D_{1} L^{2}}\right]^{\frac{1}{q}}
\end{aligned}
$$

If $H \ll W_{f}$

$$
W_{f}=\frac{V_{1} L}{2}\left(\frac{12 \rho}{\sigma_{0}} \frac{\alpha^{2}}{L^{4}}\right)^{1 / 2}
$$




$$
n=1+\left[\frac{\alpha V_{1}^{2}}{3 \sqrt{2} D L^{3}}\left(\frac{12 \rho}{\sigma_{0}}\right)^{\frac{1}{2}}\right]^{\frac{1}{q}}
$$

\section{Scaling effects on dynamic response}

The importance of deriving the dimensionless numbers of the system to avoid repetitive experiments has already been discussed in section 'Introduction'. In the case of blast loading, the response number $R_{n}$, defined by equation (74), is often used in dimensional analysis. Indeed, as depicted in equation (75), the expression of the transverse displacement is a linear function of $R_{n}$. However, in the case of localised blast, both the shape $\left(\alpha / L^{2}\right)$ and the amplitude $\left(p_{0}\right)$ of the load enter in the expression of the normalised displacement. The influence of these parameters is illustrated in Figure 8. Clearly, the normalised displacement will increase with the increase in either the shape factor or the ratio $\eta$. The chart of Figure 8(a) was obtained for a constant shape factor equal to 0.44, while the data displayed in Figure 8(b) correspond to a constant ratio $\eta$ of 6 . In each case, the $\eta$ values were previously compared to the corresponding $\eta_{\text {crit }}$ for the case of simply supported beam to determine the corresponding analytical formula for $W_{f}$ to be used (i.e. equation (19) or (40))

$$
\begin{gathered}
R_{n}=\frac{p_{0}^{2} \tau^{2}}{\rho \sigma_{0} H^{2}}\left(\frac{L}{H}\right)^{2} \\
\frac{W_{f}}{H}=R_{n}\left[\frac{\alpha}{L^{2}}\left(1-\frac{1}{2 \eta}\right)-\frac{1}{6}\right]
\end{gathered}
$$

\section{FE analyses}

In this section, the accuracy of the analytical models is validated by corresponding FE models set up in ABAQUS 6.14 ${ }^{\circledR}$. The FE model was idealised with one-dimensional (1D) planar

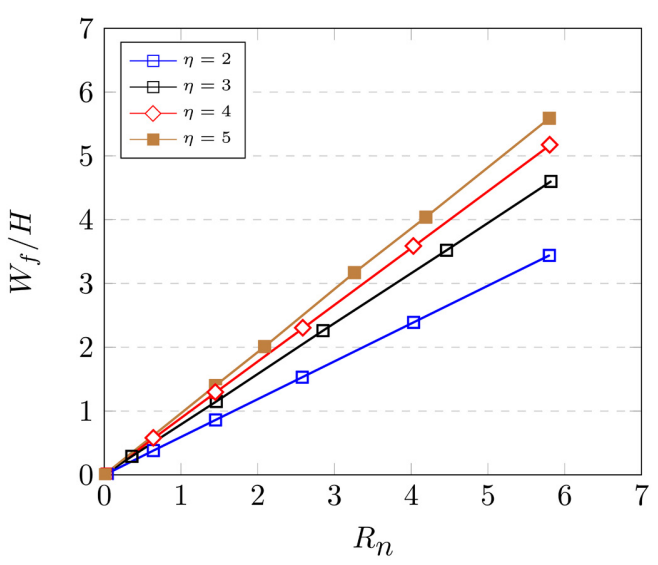

(a)

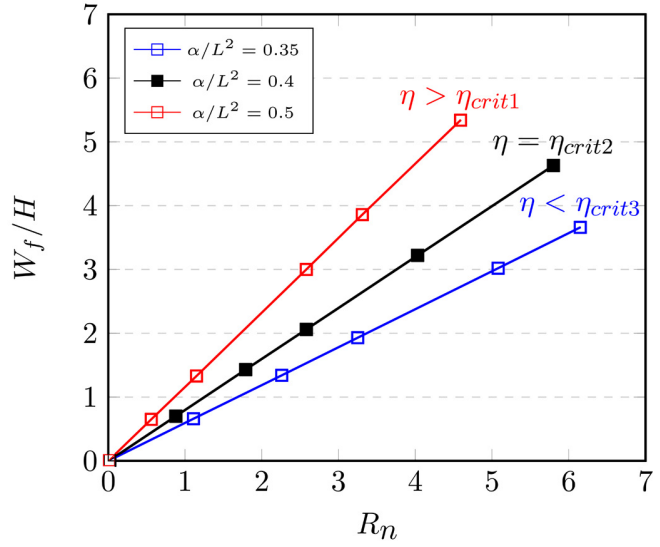

(b)

Figure 8. Influence of the (a) ratio $\eta$ and (b) load shape factor $\alpha / L^{2}$ on maximum deflection. 
Table 2. FE model parameters.

\begin{tabular}{llllll}
\hline Material properties & \multicolumn{2}{l}{ Dimensions $(\mathrm{mm})$} & \multicolumn{2}{l}{ Loading } \\
\hline$\rho\left(\right.$ tonne $\left.\cdot \mathrm{mm}^{-3}\right)$ & $7.85 \times 10^{-9}$ & $B$ & 100 & $p_{0}$ & 40 \\
$E(\mathrm{MPa})$ & $2.1 \times 10^{5}$ & $H$ & 100 & $b$ & -0.01 \\
$\sigma_{0}(\mathrm{MPa})$ & 250 & $2 L$ & 1000 & $\tau(\mathrm{s})$ & 0.001 \\
\hline
\end{tabular}

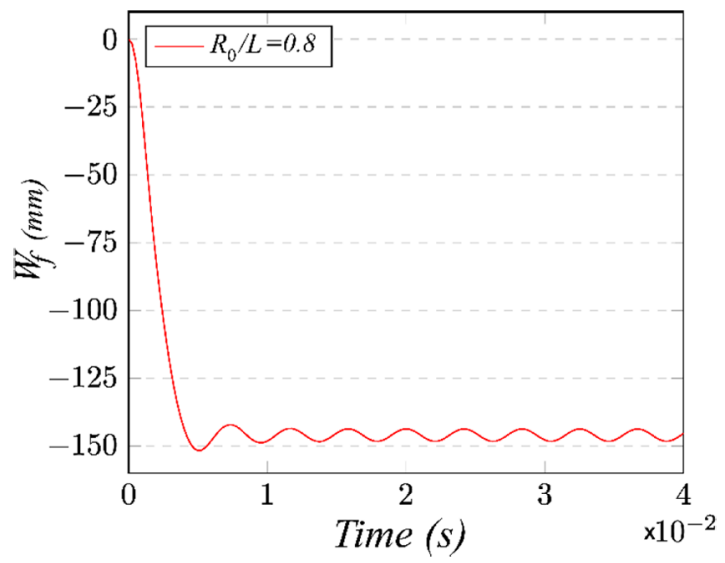

Figure 9. Transverse displacement at midspan (bending only).

geometry, meshed with two-noded beam elements and had elastic-perfectly plastic material behaviour. The beam's material properties and the dimensions are presented in Table 2. The FE model was discretised with 200 1D linear beam elements B21 and pinned at the edges, encompassing a total of 600 degrees-of-freedom.

The loading was generated as an analytical field and applied directly on the top of the beam. The field was considered with the profile as in Figure 1 (with uniform distribution over the length of $R_{0}$ from the midspan and exponentially decaying beyond $R_{0}$ ). The assumed pulse duration and magnitude was to ensure the beam has reached the 'residual oscillation regime' displayed in Figure 9. The beam was restricted to only deflect transversely and was simply supported at the edges.

The preliminary results presented in Figures 9 to 13 considered the bending effects only. The results of Figure 10 were used to discern the critical load ratio $\eta_{\text {crit }}$ that triggers the apparition of the travelling plastic hinges. Therefore, the two analytical expressions of $W_{f} / L$, corresponding to the cases $\eta \leq \eta_{\text {crit }}$ and $\eta \geq \eta_{\text {crit }}$, are plotted as functions of $R_{0} / L$. At constant $p_{0}$, when the radius of the central zone expands, $\eta$ increases while $\eta_{c r i t}$ decreases (since the loading parameter $\alpha$ increases in equations (14) and (22)). Hence, ABAQUS results logically approach the first case $\left(\eta<\eta_{\text {crit }}\right)$ for small values of $R_{0}$ and the second case for higher values. At $R_{0} / L=0.4$, a transition in the deformation pattern occurs, which is observed in Figure 10 and corresponds to the critical load factor (i.e. the configuration where $\left|\eta-\eta_{\text {crit }}\right|=0$ ) in Table 3. 


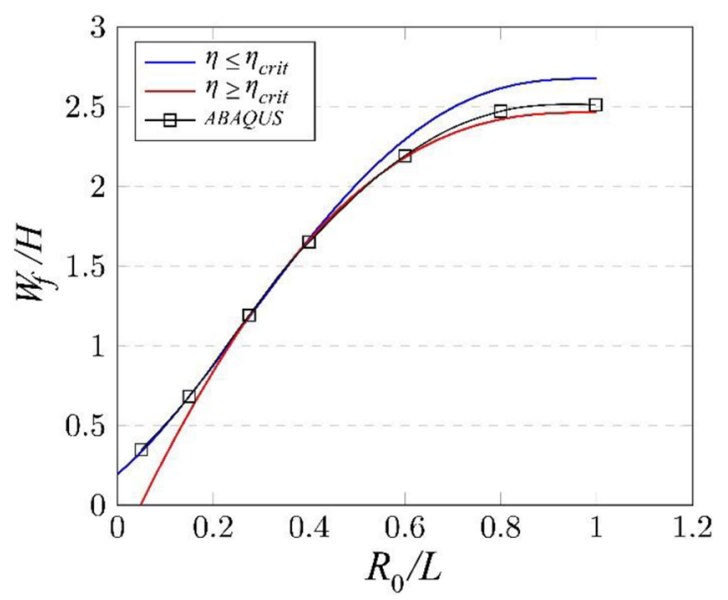

Figure 10. Confirmation of a turning point $\eta_{\text {crit }}$ in beams' response to blast loading (bending only).

Table 3. Variation of $\eta$ and $\eta_{\text {crit }}$ for different values of $R_{0} / L$.

\begin{tabular}{llllllll}
\hline$R_{0} / L$ & 0.05 & 0.15 & 0.275 & 0.4 & 0.6 & 0.8 & 1.00 \\
$\eta$ & 3.19 & 4.31 & 5.49 & 6.43 & 7.45 & 7.92 & 8.00 \\
$\eta_{\text {crit }}$ & $\infty$ & $\infty$ & 34.60 & 5.85 & 3.52 & 3.07 & 3.00 \\
\hline
\end{tabular}

The numerical results of the equivalent plastic strain for the two cases of $R_{0} / L=0.4$ and $R_{0} / L=0.8$ are plotted at $t=\tau$ in Figure 12. The peaks observed on either side of the midspan confirm the initial position of the plastic hinge $\xi_{0}$. As expected, the higher the $R_{0} / L$ ratio, the higher the value of $\xi_{0}$. Furthermore, when $R_{0} / L$ decreases to values below 0.4 , corresponding to the case of $\eta \leq \eta_{\text {crit }}$, the peaks are no longer visible because there is only one unique static hinge occurring at the midspan. The appearance of the incipient plastic hinges and the position of the travelling plastic hinges at various times are illustrated in Figure 11, appearing as region with maximum equivalent plastic strain. The equivalent plastic strain, $\bar{\varepsilon}_{p}$, is the time integral of the equivalent plastic strain rate $\dot{\bar{\varepsilon}}_{p}$, where $\dot{\bar{\varepsilon}}_{p}=\sqrt{\frac{2}{3} \dot{\varepsilon}_{i j}^{p} \dot{\varepsilon}_{i j}^{p}} \dot{\bar{\varepsilon}}_{p}=\sqrt{\frac{2}{3} \dot{\varepsilon}_{i j}^{p} \dot{\varepsilon}_{i j}^{p}}$ and $\dot{\varepsilon}_{i j}^{p}$ is the plastic strain-rate tensor.

Figure 13 compares the predicted numerical and analytical values of the position of incipient plastic hinges for various loading conditions. It is recognised that for all loading radii, the length of the plastic hinge (the ordinate in Figure 13) is smaller than the abscissa, confirming the hypothesis $\xi_{0} \leq R_{0}$ made in the previous section. Clearly, both the numerical and analytical results converge to the same value as the loading radius increases, which would also correspond to the condition of $\eta \gg \eta_{\text {crit }}$. Consequently, the end time $T_{2}$ predicted numerically converges to that evaluated theoretically, as shown in Figure 14. However, the difference between the calculated $T_{2}$ and $\xi_{0}$ and those of numerically predicted increases when the loading is more localised at the centre, that is, when $R_{0} / L<0.5$.

One reason that could account for this difference is the fact that the analytical model considers the plastic hinges as points, while the peaks of plastic strain measured in ABAQUS 


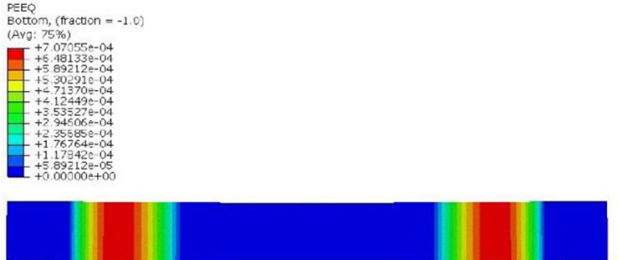

(a)

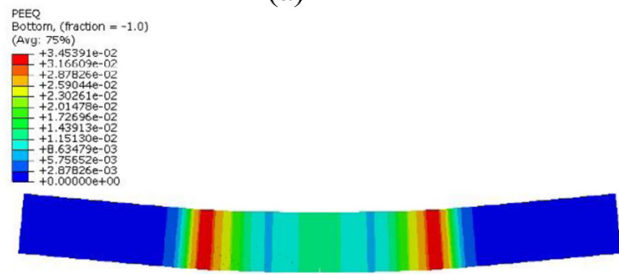

(b)
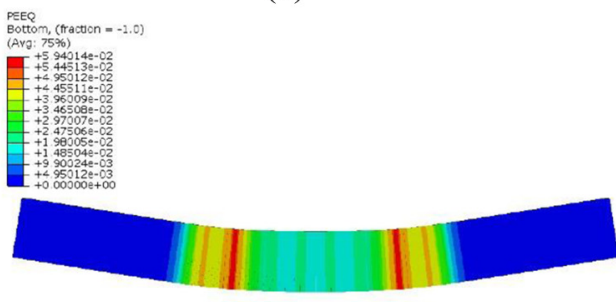

(c)
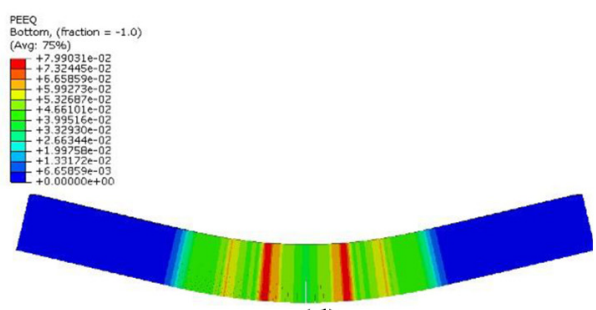

(d)

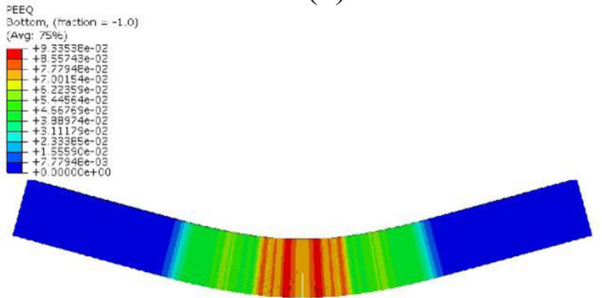

(e)

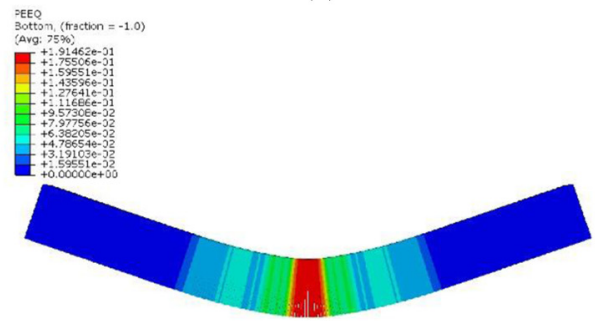

(f)

Figure I I. Distribution of the equivalent plastic strain $\left(\bar{\varepsilon}_{p}\right)$ at various times (depth of the beam is shown as a visual aid): (a) $t=0.3 \mathrm{~ms}$, (b) $t=1 \mathrm{~ms}$, (c) $t=1.5 \mathrm{~ms}$, (d) $t=2.1 \mathrm{~ms}$, (e) $t=2.4 \mathrm{~ms}$ and (f) $t=3 \mathrm{~ms}$.

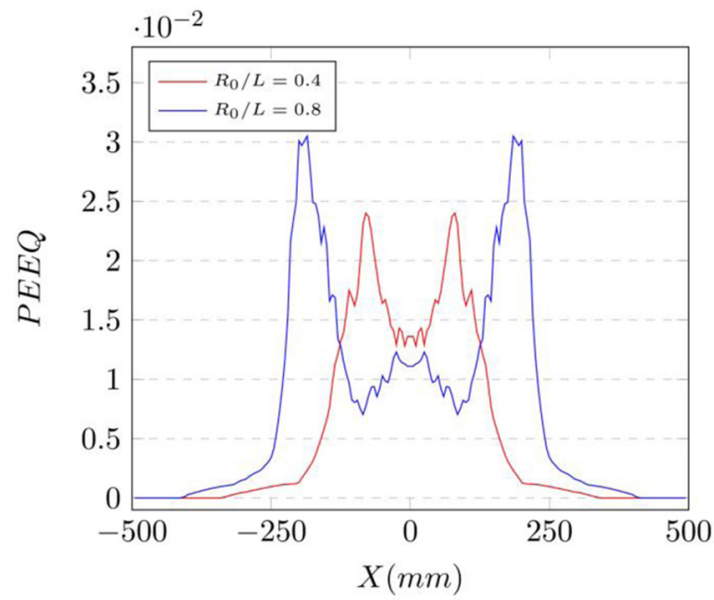

Figure 12. PEEQ distribution revealing the position of the plastic hinges along the beam (bending only). 


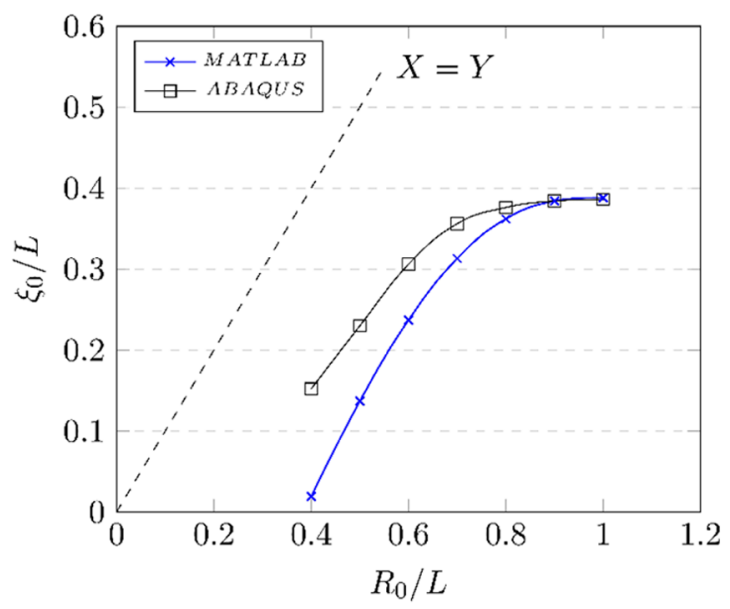

Figure 13. Initial position of the plastic hinges for different values of $R_{0} / L$.

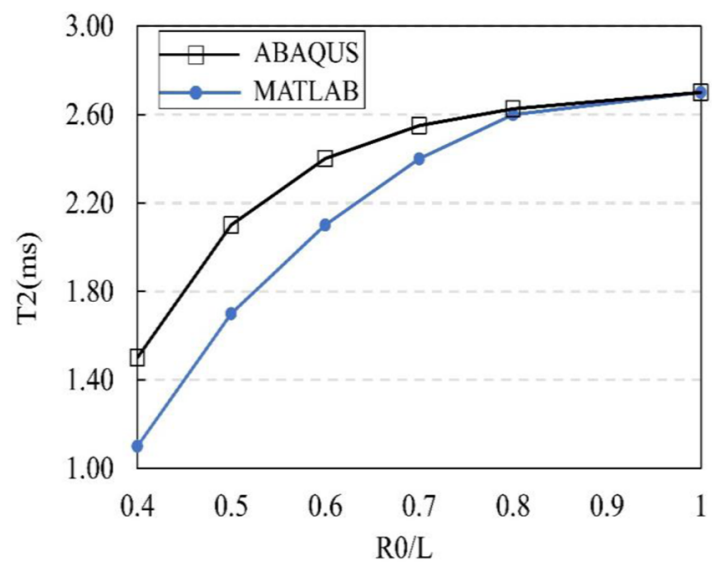

Figure 14. End time of central hinge motion for different values of $R_{0} / L$.

always possess a certain width. We verify the approximate location of the plastic hinge by recording the average coordinates where the maxima of the equivalent plastic strains $\varepsilon_{p}$ would occur, and plot the results in Figure 15. The results demonstrate strong correlation when the loading distribution is uniform, nevertheless, similar to the observations in Figure 13, the difference increases to more than $20 \%$ as the loading radii decreases to less than half the side length. In such beams, in addition to either side of the beam, an incipient maximum of $\varepsilon_{p}$ would occur at the centre which remains stationery. Furthermore, the side plastic hinges do not necessarily reach the centre as the plasticity propagates through the beam and they rest at a close distance from the midspan after time $T_{2}$ (numerical results on the case of $R_{0} / L=0.4$ and $R_{0} / L=0.5$ in Figure 15). This is an interesting observation that is also 


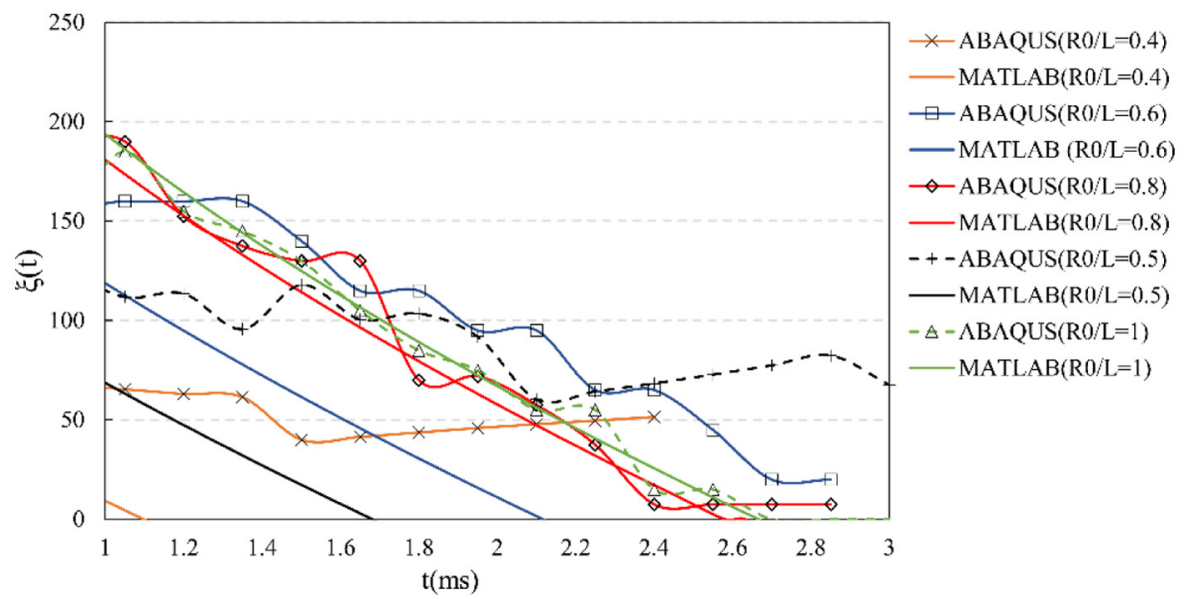

Figure I5. Variations in the length of plastic hinge $\xi(t)$ in time for various loading radii.

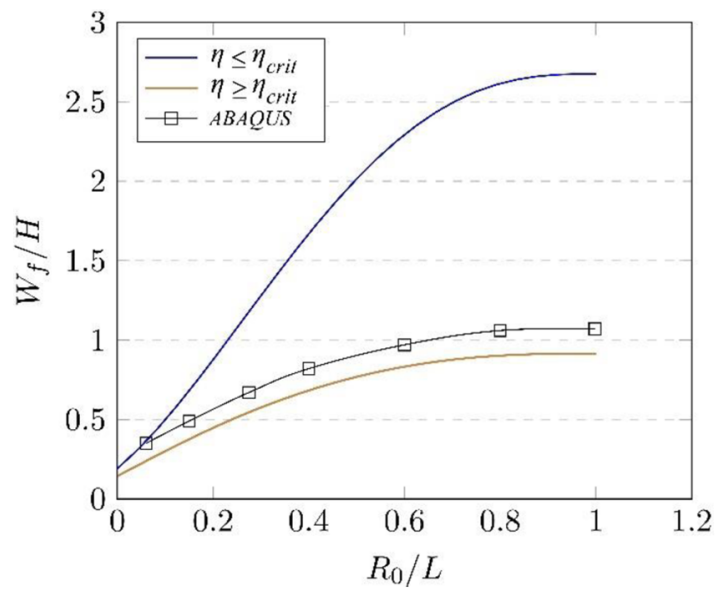

Figure 16. Influence of the membrane effect on the beam's transverse displacement.

pertinent with the case of plates and corroborates with experimental results of Jacob et al. (2007) and Langdon et al. (2015) as the nature of localised blast loads due to the proximal charges is perceived to be significantly different from more uniform loading types, wherein the permanent deformation profile of locally blasted structure is described as a small dome atop the larger dome. It should nevertheless be appreciated that the actual response of the beam under blast is an intrinsically complex process which cannot be precisely modelled with analytical simplifications.

The membrane forces are only involved in the behaviour of axially restrained beams. To investigate the membrane effects numerically, an FE model was set up with pinned boundary condition and permitted for geometric nonlinearities. Figure 16 compares the numerical 


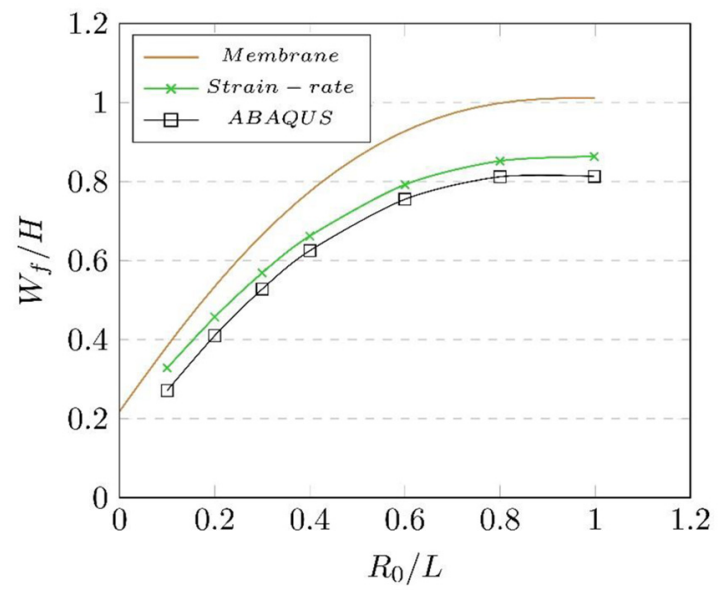

Figure 17. Influence of the strain-rate effect on the beam's transverse displacement, with $D_{1}=40.4 \mathrm{~s}^{-1}$ and $q=5$.

results with the analytical expressions obtained with and without the membrane effects. As expected, the predicted results without the membrane effect overestimate the beam's final plastic deflection, while the analysis with membrane effects gives conservative estimates. This conservative estimation is expected, since the results evaluated with assumed square yield conditions $M=M_{0}$ and $N=N_{0}$ overestimate the beam's resistance and give rise to the deviation of the curve from numerical results. Similarly, there is a good correlation between the analytical and numerical results considering the influence of the strain-rate effect in material constitutive model as in Figure 17.

The transverse displacement and stress at loading time $\tau$ and loading duration time $t=T$ are plotted in Figure 18.

In the previous figures, the curves were mainly displayed as functions of $R_{0}$. As stated previously, the dynamic load factor $\eta$ reduces as $R_{0}$ decreases. Evidently, provided the maximum pressure $p_{0}$ is kept constant, the smaller the values of $R_{0}$, the smaller the global amount of energy transmitted to the beam. Hence, to avoid the decrease in $\eta$ with $R_{0}$, the analysis in Figure 19 was conducted at constant $I_{c}$, with $I_{c}$ being Youngdahl's effective impulse defined by equation (76). Hence, for a constant impulse, the concentrated distribution of load was more damaging than the uniform pressure

$$
\begin{gathered}
I_{c}=\int_{t_{i}}^{t_{f}} P(t) d t \\
I_{c}=\frac{p_{0} \tau}{L}\left[R_{0}+\frac{a}{b}\left(e^{b L}-e^{b R_{0}}\right)\right]
\end{gathered}
$$

\section{Conclusion}

This article deals with a theoretical model within the framework of rigid plasticity to predict the response of ductile metallic beam subjected to localised blast load, using the upper bound 


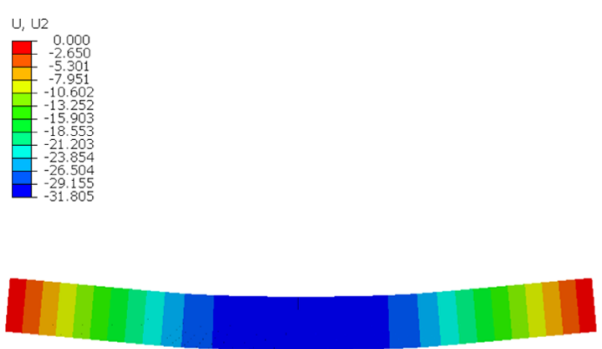

(a)

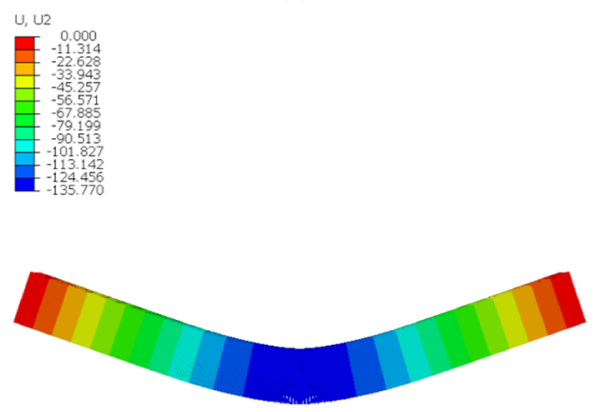

(b)
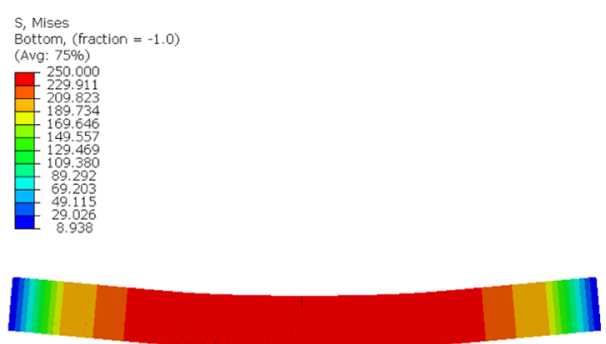

(c)

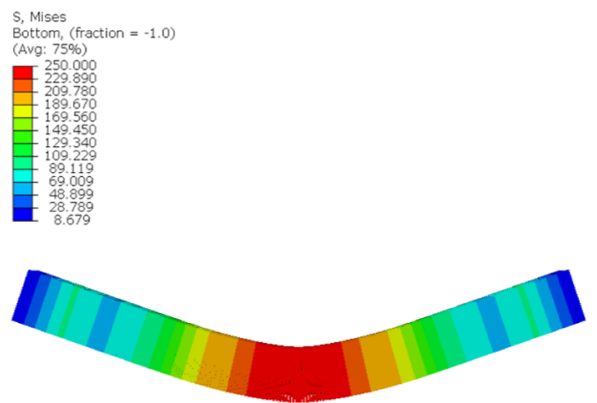

(d)

Figure 18. (a, b) Distribution of the displacement at $t=\tau$ and final time $t=3 \mathrm{~ms}$; (c, d) stress at $t=\tau$ and final time $t=3 \mathrm{~ms}\left(R_{0} / L=0.8\right)$.

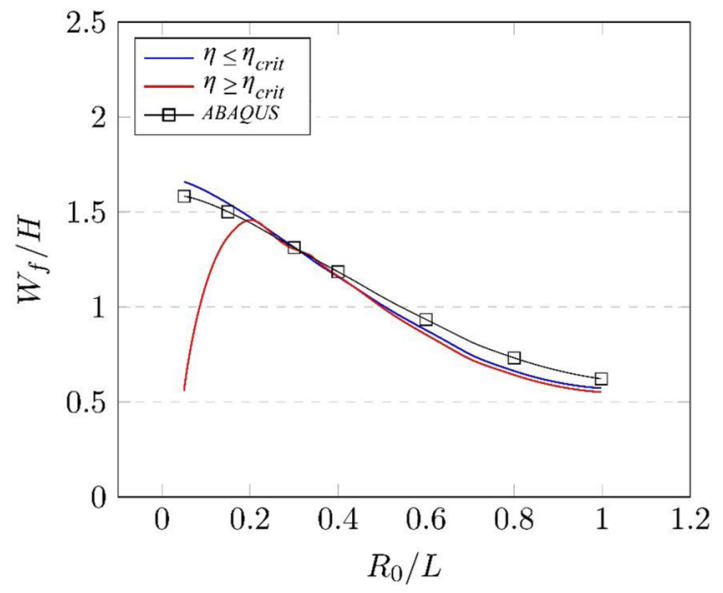

Figure 19. Influence of the load shape on deflection for a constant effective impulse $l_{\mathrm{e}}=0.02$ and various values of $p_{0}$. 
and lower bound theorems of limit analysis. Assuming a piecewise continuous loading function formerly studied by some researchers (Karagiozova et al., 2010; Micallef et al., 2012), the exact static plastic collapse pressure was determined. The analysis was extended to the dynamic case by considering Johansen's interactive yield curves where the phenomena of bending moments, transverse shear and membrane forces control the plastic flow. A study on the response of strain-rate-sensitive beams to localised blasts and the influence of pulse shape were also discussed.

In the case of bending moment dominated beams (shallow beams), the incipient velocity profile was governed by the travelling plastic hinges in three stages of the analysis. A critical load factor which triggers the apparition of the travelling plastic hinges was identified, leading to two different expressions for the transverse displacements. In the case of the transverse shear and membrane effects dominated beams (deep beams and thin beams, respectively), the transverse deformations were obtained for the impulsive blast loads by considering the simplified interactive yield curves representing each phenomenon.

A 1D beam numerical model was set up in ABAQUS to validate the analytical results and highlighted the different assumptions that were made in the calculations. The numerical results confirmed the initial position of the plastic hinges to be always restrained in the central zone of blast load, which is characterised by uniform pressure $\left[-R_{0}, R_{0}\right]$. Furthermore, the assumption of a simplified circumscribing yield curve used to linearise the expression of the deflection for finite displacements was confirmed to be a slight overestimation of beam's actual resistance.

As a final remark, it must be mentioned that preliminary studies showed that the effect of rotatory inertia is far less important than that of shear strain and thus the former has not been included in the studies conducted on deep beams. Strong correlation between the results obtained by the proposed analytical model and the FE simulations show the correctness and accuracy of this assumption.

\section{Declaration of conflicting interests}

The author(s) declared no potential conflicts of interest with respect to the research, authorship and/or publication of this article.

\section{Funding}

The author(s) received no financial support for the research, authorship and/or publication of this article.

\section{References}

Bonorchis D and Nurick GN (2007) The effect of welded boundaries on the response of rectangular hot-rolled mild steel plates subjected to localised blast loading. International Journal of Impact Engineering 34: 1729-1738.

Bonorchis D and Nurick GN (2009) The influence of boundary conditions on the loading of rectangular plates subjected to localised blast loading - importance in numerical simulations. International Journal of Impact Engineering 36: 40-52.

Chung Kim Yuen S, Langdon GS, Nurick GN, et al. (2012) Response of V-shape plates to localised blast load: experiments and numerical simulation. International Journal of Impact Engineering 46: 97-109. 
Fallah AS and Louca LA (2007) Pressure-impulse diagrams for elastic-plastic-hardening and softening single-degree-of-freedom models subjected to blast loading. International Journal of Impact Engineering 34: 823-842.

Fleck NA and Deshpande VS (2004) The resistance of clamped sandwich beams to shock loading. Journal of Applied Mechanics 71: 386-401.

Hopkins HG and Prager W (1953) The load carrying capacity of circular plates. Journal of the Mechanics and Physics of Solids 2: 1-18.

Jacob N, Nurick GN and Langdon GS (2007) The effect of stand-off distance on the failure of fully clamped circular mild steel plates subjected to blast loads. Engineering Structures 29: 2723-2736.

Jacob N, Yuen SCK, Nurick GN, et al. (2004) Scaling aspects of quadrangular plates subjected to localised blast loads - experiments and predictions. International Journal of Impact Engineering 30: $1179-1208$.

Jones N (1971) A theoretical study of the dynamic plastic behavior of beams and plates with finitedeflections. International Journal of Solids and Structures 7: 1007-1029.

Jones N (1990) Structural Impact (1st edn). Cambridge: Cambridge University Press.

Jones N (2010) Energy-absorbing effectiveness factor. International Journal of Impact Engineering 37: $754-765$.

Jones N (2014) Dynamic inelastic response of strain rate sensitive ductile plates due to large impact, dynamic pressure and explosive loadings. International Journal of Impact Engineering 74: 3-15.

Jones N and Kim S-B (1997) A study on the large ductile deformations and perforation of mild steel plates struck by a mass - part II: discussion. Journal of Pressure Vessel Technology 119: 185-191.

Jones N and Walters R (1983) Large deflections of rectangular plates. Journal of Ship Research 18: $125-131$.

Karagiozova D, Langdon GS, Nurick GN, et al. (2010) Simulation of the response of fibre-metal laminates to localised blast loading. International Journal of Impact Engineering 37: 766-782.

Karagiozova D, Yu TX and Lu G (2013) Transverse blast loading of hollow beams with square crosssections. Thin-Walled Structures 62: 169-178.

Langdon GS, Lee WC and Louca LA (2015) The influence of material type on the response of plates to air-blast loading. International Journal of Impact Engineering 78: 150-160.

Larcher M and Casadei F (2010) Explosions in complex geometries - a comparison of several approaches. International Journal of Protective Structures 1: 169-195.

Ma GW, Huang X and Li JC (2010) Simplified damage assessment method for buried structures against external blast load. Journal of Structural Engineering 136: 603-612.

Micallef K (2013) The dynamic response of blast-loaded monolithic and composite plated structures. PhD Thesis, Imperial College London, London.

Micallef K, Fallah AS, Pope DJ, et al. (2012) The dynamic performance of simply-supported rigidplastic circular steel plates subjected to localised blast loading. International Journal of Mechanical Sciences 65: 177-191.

Micallef K, Fallah AS, Pope DJ, et al. (2015) On dimensionless loading parameters for close-in blasts. The International Journal of Multiphysics 9: 171-194.

Nurick GN and Balden V (2010) Localised blast loaded circular plates: an experimental and numerical investigation. In: Proceedings of the IMPLAST 2010 conference, Providence, RI, 12-14 October, pp. 145-152. Bethel, CT: Society for Experimental Mechanics.

Perrone N and Bhadra P (1979) A simplified method to account for plastic rate sensitivity with large deformations. Journal of Applied Mechanics 46: 811-816.

Symonds PS and Jones N (1972) Impulsive loading of fully clamped beams with finite plastic deflections and strain-rate sensitivity. International Journal of Mechanical Sciences 14: 49-69.

Symonds PS and Yu TX (1985) Counterintuitive behavior in a problem of elastic-plastic beam dynamics. Journal of Applied Mechanics 52: 517-522.

Taylor GI (1963) The pressure and impulse of submarine explosion waves on plates. In: The Scientific Papers of Sir Geoffrey Ingram Taylor, vol. 3. Cambridge: Cambridge University Press, pp. 287-303. 
Webster G (2013) Mine Kafon: the low-tech, high-design tumbleweed minesweeper. CNN. Available at: https://edition.cnn.com/2012/11/29/tech/gallery/mine-kafon-tumbleweed-minesweeper/index.html

Wierzbicki T and Nurick GN (1996) Large deformation of thin plates under localised impulsive loading. International Journal of Impact Engineering 18: 899-918.

Youngdahl CK (1971) Influence of pulse shape on the final plastic deformation of a circular plate. International Journal of Solids and Structures 7: 1127-1142.

Yu TX and Chen F (1992) The large deflection dynamic plastic response of rectangular plates. International Journal of Impact Engineering 12: 605-616.

Yuen SCK and Nurick GN (2001) Deformation and tearing of uniformly blast-loaded quadrangular stiffened plates. In: Zingoni A (ed.) Structural Engineering, Mechanics and Computation. Oxford: Elsevier Science, pp. 1029-1036.

Yuen SCK and Nurick GN (2005) Experimental and numerical studies on the response of quadrangular stiffened plates. Part I: subjected to uniform blast load. International Journal of Impact Engineering 31: 55-83.

\section{Appendix I}

\section{Notation}

$\begin{array}{ll}a & \text { loading parameter, }(1) \\ b & \text { loading exponent }\left(L^{-1}\right) \\ m_{b} & \text { mass of the beam }(M) \\ p_{0} & \text { maximum overpressure }\left(M L^{-1} T^{-2}\right) \\ p_{c} & \text { static collapse pressure }\left(M L^{-1} T^{-2}\right) \\ p(x) & \text { spatial part of pressure pulse load }\left(M L^{-1} T^{-2}\right) \\ p^{*}(t) & \text { temporal part of pressure pulse load }(1) \\ q & \text { strain-rate exponent }(1) \\ w_{i} & \text { transverse displacement at the } i \text { th phase }(L) \\ \dot{w}_{i} & \text { transverse velocity at the } i \text { th phase }\left(L T^{-1}\right) \\ \ddot{w}_{i} & \text { transverse acceleration at the } i \text { th phase }\left(L T^{-2}\right) \\ w_{f} & \text { final transverse displacement }(L) \\ A-F & \text { integration constants }(\text { various }) \\ \bar{B} & \text { beam width }(L) \\ D_{1} & \text { strain-rate coefficient }\left(T^{-1}\right) \\ H & \text { thickness of the beam }(L) \\ M_{0} & \text { maximum plastic bending moment }\left(M L^{2} T^{-2}\right) \\ N_{0} & \text { maximum membrane force }\left(M L T^{-2}\right) \\ N & \text { plastic membrane force }\left(M L T^{-2}\right) \\ Q_{0} & \text { maximum transverse shear force }\left(M L T^{-2}\right) \\ R_{0} & \text { radius of central uniformly loaded region }(L) \\ V_{1} & \text { impulsive velocity of uniform load }\left(L T^{-1}\right) \\ V_{0} & \text { central transverse displacement }[L] \\ W & \text { impulsive velocity of localised load }\left(L T^{-1}\right) \\ \alpha & \text { lower bound static collapse coefficient }\left(L^{3}\right) \\ \beta & \text { upper bound static collapse coefficient }\left(L^{3}\right) \\ \dot{\varepsilon} & \text { strain rate }(1) \\ \eta & \text { plastic hinge generalised coordinate }(1) \\ & \text { dynamic load factor }(1)\end{array}$




$\begin{array}{ll}\eta_{c r i t} & \text { critical dynamic load factor }(1) \\ \dot{\kappa} & \text { curvature rate }\left(T^{-1}\right) \\ \lambda & \text { dimensionless kinetic energy }(1) \\ \mu & \text { areal density }(\rho H),\left(M L^{-2}\right) \\ \rho & \text { material density }\left(M L^{-2}\right) \\ \tau & \text { duration of the pulse }(\mathrm{T}) \\ \sigma_{0} & \text { static yield stress }\left(M L T^{-2}\right) \\ \sigma_{0}^{\prime} & \text { dynamic flow stress }\left(M L T^{-2}\right) \\ \dot{\theta}_{i} & \text { rotational velocity in direction of } i,\left(T^{-1}\right)\end{array}$

